\title{
Alternative oxidase gene induced by nitric oxide is involved in the regulation of ROS and enhances the resistance of Pleurotus ostreatus to heat stress
}

\author{
Ludan Hou ${ }^{1,2}$, Mengran Zhao ${ }^{1,2}$, Chenyang Huang ${ }^{1,2}$, Qi He ${ }^{1,2,3}$, Lijiao Zhang ${ }^{1,2}$ and Jinxia Zhang ${ }^{1,2^{*}}$ (D)
}

\begin{abstract}
Background: In China, during the cultivation process of Pleurotus ostreatus, the yield and quality of fruiting bodies are easily affected by high temperatures in summer. Nitric oxide (NO) plays an important regulatory role in the response to abiotic stress, and previous studies have found that NO can induce alternative oxidase (aox) experssion in response to heat stress (HS) by regulating aconitase. However, the regulatory pathway of NO is complex, and the function and regulation of the aox gene in the response to HS remain unclear.
\end{abstract}

Results: In this study, we found that $\mathrm{NO}$ affected nicotinamide adenine dinucleotide (NADH) and adenosine triphosphate (ATP) levels, reduced hydrogen peroxide $\left(\mathrm{H}_{2} \mathrm{O}_{2}\right)$ and superoxide anion $\left(\mathrm{O}_{2}{ }^{-}\right)$contents, and slowed $\mathrm{O}_{2}{ }^{-}$production. Further RNA-Seq results showed that NO regulated the oxidation-reduction process and oxidoreductase activity, affected the cellular respiration pathway and activated aox gene expression. The function of aox was determined by constructing overexpression (OE) and RNA interference (RNAi) strains. The results showed that the OE-aox strains exhibited obviously improved growth recovery after exposure to HS. During exposure to HS, the OE-aox strains exhibited reduced levels of NADH, the product of the tricarboxylic acid (TCA) cycle, and decreased synthesis of ATP, which reduced the production and accumulation of reactive oxygen species (ROS), whereas the RNAi-aox strains exhibited the opposite result. In addition, aox mediated the expression of antioxidant enzyme genes in the mycelia of P. ostreatus under HS through the retrograde signaling pathway.

Conclusions: This study shows that the expression of the aox gene in P. ostreatus mycelia can be induced by NO under HS, that it regulates the TCA cycle and cell respiration to reduce the production of ROS, and that it can mediate the retrograde signaling pathway involved in the mycelial response to HS.

Keywords: Pleurotus ostreatus, Nitric oxide, ROS, RNA-Seq, Alternative oxidase, Antioxidant enzymes

*Correspondence: zhangjinxia@caas.cn

${ }^{2}$ Key Laboratory of Microbial Resources, Ministry of Agriculture and Rural Affairs, 10081 Beijing, China

Full list of author information is available at the end of the article

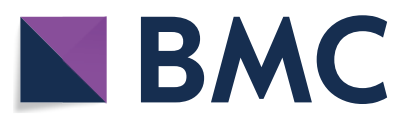

(c) The Author(s) 2021. Open Access This article is licensed under a Creative Commons Attribution 4.0 International License, which permits use, sharing, adaptation, distribution and reproduction in any medium or format, as long as you give appropriate credit to the original author(s) and the source, provide a link to the Creative Commons licence, and indicate if changes were made. The images or other third party material in this article are included in the article's Creative Commons licence, unless indicated otherwise in a credit line to the material. If material is not included in the article's Creative Commons licence and your intended use is not permitted by statutory regulation or exceeds the permitted use, you will need to obtain permission directly from the copyright holder. To view a copy of this licence, visit http://creativecommons.org/licenses/by/4.0/. The Creative Commons Public Domain Dedication waiver (http://creativecommons.org/ publicdomain/zero/1.0/) applies to the data made available in this article, unless otherwise stated in a credit line to the data. 


\section{Background}

Pleurotus ostreatus is one of the most widely cultivated mushroom species globally and is mainly cultivated in horticultural facilities in China [1]. During the cultivation process of $P$. ostreatus, high temperatures in summer limit the growth of mycelia, the formation of primordia and the development of fruiting bodies, and thereby seriously affecting the yield. However, the mycelia response to changes in temperature is very complex and includes different physiological and metabolic changes that affect the overall development of edible fungi as well as interactions between cells and molecules. Metabolomics analyses have shown that heat stress (HS) promotes the degradation of unsaturated fatty acids and nucleotides, increases the contents of amino acids and vitamins, and accelerates glycolysis and the tricarboxylic acid (TCA) cycle [2]. Furthermore, reactive oxygen species (ROS) accumulate in the mycelium during exposure to HS, which results in cell damage and even apoptosis [3]. The synthesis of trehalose and heat shock proteins is an important component of the response of edible fungi to high temperatures and to their resistance to heat [4]. Moreover, nitric oxide (NO) and calcium $\left(\mathrm{Ca}^{2+}\right)$ as signaling molecules, also play an active protective role during exposure to HS [5]. In recent years, increasing attention has been given to the mechanism through which fungi respond to $\mathrm{HS}$.

$\mathrm{NO}$, an essential endogenous signaling molecule that is involved in many biological processes in plants, animals, bacteria, and fungi, is considered a broad-spectrum antistress molecule [6-8]. The role of $\mathrm{NO}$ as a signaling molecule in the growth, development and stress responses of plants has been widely studied and has become the focus of much research. Previous studies have revealed that $\mathrm{NO}$ contributes to various aspects of stress tolerances by influencing ROS metabolism [9]. $\mathrm{Ca}^{2+}$-mediated NO signal transduction is involved in the response of Brassica seedlings to metalloid stress [10], and in Prunus persica (L.) Batsch, treatment with a NO solution can protect the fruit from pathogen infection by inducing the activity of defense enzymes and the expression of pathogenesis-related genes [11]. In two ecotypes of reeds, NO significantly reduces the contents of hydrogen peroxide $\left(\mathrm{H}_{2} \mathrm{O}_{2}\right)$ and malondialdehyde in calli and significantly inhibits the increase in ion leakage and growth inhibition and decrease in cell viability induced by HS [12]. Previous studies have shown that NO can regulate ROS content in two ways: by acting as a free radical to neutralize ROS or by acting as a signaling molecule to initiate gene expression via a molecular cascade [13]. In fungi, the function of $\mathrm{NO}$ has been gradually studied in recent years. For example, during fungal development, NO might not only induce sexual development
[14] but it may also be involved in conidiation, spore germination, and formation of the parasitic appressoria structure, among other processes $[15,16]$. In addition, studies have shown that $\mathrm{NO}$ can participate in the regulation of secondary metabolism in fungi. For example, NO can stimulate the activities of phenylalanine ammonia lyase and chalcone synthase in fruiting bodies to induce the accumulation of phenols and quinolines [17]. In addition, NO participates in the response pathway of fungi to various environmental stresses $[18,19]$. Recently, NO has also been found in edible mushroom. Specifically, in Ganoderma lucidum, NO is involved in the regulation of ganoderic acid synthesis [5], and in P. ostreatus, NO can alleviate the oxidative damage induced in mycelia by HS [20]. However, the function and regulation of $\mathrm{NO}$ in fungi is less clear than in plants.

Mitochondria are the main site of ROS bursts and play a key role in cell energy metabolism. The electron transport chain (ETC) of mitochondria consists of two pathways: the main cytochrome $\mathrm{c}$ pathway and the alternative pathway. Alternative oxidase (AOX, encoded by aox), an integral protein $(32-36 \mathrm{kDa})$ of the inner mitochondrial membrane [21], is a component of the mitochondrial respiratory pathway that is widely found in higher plants and some fungi and algae and is responsible for the activity of the alternative respiratory pathway [22]. In Neurospora crassa, a model fungal organism, aox transcripts are undetectable or present at very low levels under normal growth conditions [23]. However, when cells are grown in the presence of drugs that inhibit the cytochromemediated electron transport chain, such as antimycin A, which inhibits complex III, expression of the aox gene is strongly induced and the AOX protein can be found in mitochondria [24]. In addition, AOX is also present in mutant strains that lack components of the cytochromemediated electron transport chain [25]. Recently, the function of AOX has been widely studied. In plants, the AOX pathway plays an important role in maintaining metabolism and signal homeostasis during exposure to abiotic and biotic stress [26]. One of the possible functions of AOX is regulating the production of $\operatorname{ROS}[27,28]$. The electron transport flow generated by AOX bypasses proton pump complexes III and IV, which affects adenosine triphosphate (ATP) production and reduces electron leakage and ROS production [29]. In tobacco, AOX can maintain plant environmental homeostasis and enhance the tolerance of cells to drought stress by controlling the respiration rate, photosynthesis and chlorophyll synthesis [30]. In Medicago truncatula, NO can induce the expression of aox, and AOX can help regulate the accumulation of ROS, protect the photosystem, and enhance plant resistance to salt stress [31]. In addition, AOX enhances the tolerance of spring wheat to HS [32]. The function of 
AOX in fungi has also been reported. For instance, AOX is a determinant for growth and sporulation in the early diverging fungus Blastocladiella emersonii [33] and in Aspergillus fumigatus, silencing of the mitochondrial aox gene makes the strain more sensitive to ROS, and easier to kill by macrophages [34]. However, the function of AOX in P. ostreatus remains unclear.

The expression of the aox gene is also induced by a wide range of biotic and abiotic stresses [35], and previous studies have shown that upreglation of the aox gene under stress conditions is associated with stress-dependent ROS production in tobacco [36]. Our previous study revealed that NO, as a signaling molecule under HS, can increase the citric acid content by inhibiting aconitase in P. ostreatus. The accumulation of citric acid can induce the expression of the aox gene and enhance the resistance of mycelia to HS [20]. However, the regulatory pathway through which NO alleviates mycelial stress is complex, and the mechanism through which the aox gene of $P$. ostreatus enhances the thermostability of mycelia remains unclear; hence, further research is needed to obtain a more in-depth understanding of this phenomenon. In this study, RNA sequencing (RNA-Seq) technology was used to explore the possible regulatory pathways and key genes involved in the NO-mediated alleviation of HS-induced damage in P. ostreatus, and the regulatory effect of $\mathrm{NO}$ on the expression of the aox gene was studied in detail. Moreover, the relationships among aox, energy metabolism and ROS were explored using overexpression (OE) and RNA interference (RNAi) technology.

\section{Results}

Exogenous NO affected the energy metabolism of mycelia and reduced the production and accumulation of ROS

$\mathrm{NO}$, an important signaling molecule, plays an important regulatory role in the growth and development of organisms and their responses to stress. Our previous study showed that NO plays an important role in the response of $P$. ostreatus to HS [20]. In this study, the addition of exogenous NO promoted the recovery of $P$. ostreatus mycelial growth after HS, as shown in Fig. 1A (the red arrow indicates regenerated mycelia after $\mathrm{HS}$ ). $\mathrm{H}_{2} \mathrm{O}_{2}$ and $\mathrm{O}_{2}{ }^{-}$are important components of ROS, and as shown in Fig. 1B, the content of $\mathrm{H}_{2} \mathrm{O}_{2}$ in mycelia increased significantly after $\mathrm{HS}$ (HS vs. control, $P=1.118 \times 10^{-6}$ ). Exogenous sodium nitroprusside (SNP, $100 \mu \mathrm{M}$ ) significantly reduced the accumulation of $\mathrm{H}_{2} \mathrm{O}_{2}$ in mycelia after HS (SNP_HS vs. HS, $P=2.430 \times 10^{-4}$ ), but the level was still significantly higher than that in the control group (SNP_HS vs. control, $P=1.864 \times 10^{-4}$ ). As shown in Figs. $1 \mathrm{C}$ and D, HS increased the $\mathrm{O}_{2}{ }^{-}$content in mycelia by 1.64-fold and the production rate of $\mathrm{O}_{2}{ }^{-}$by $60.74 \%$. In addition, compared with the levels found in the HS group, exogenous SNP decreased the $\mathrm{O}_{2}{ }^{-}$content and production rate by 12.85 and $9.34 \%$, respectively. 2-(4-carboxyphenyl)-4,4,5,5-tetramethylimidazoline1-oxyl3-oxidec (cPTIO) is an NO scavenger. Research shows that the addition of $250 \mu \mathrm{M}$ cPTIO can significantly increase the content and production rate of $\mathrm{O}_{2}{ }^{-}$. Our results showed that exogenous NO could affect not only the content of $\mathrm{H}_{2} \mathrm{O}_{2}$, but also the content and production rate of $\mathrm{O}_{2}^{-}$.

Nicotinamide adenine dinucleotide (NAD) is a coenzyme that exists in all cells and is found in two forms: oxidized $\left(\mathrm{NAD}^{+}\right)$and reduced (NADH). NADH is produced during glycolysis pathway and the TCA cycle, participates in material and energy metabolism in cells and serves as a control marker in the energy production chain of mitochondria. As shown in Fig. 1E, HS significantly increased the content of NADH in mycelia compared with the level found in the control group (HS vs. control, $P=1.500 \times 10^{-9}$ ), and this increase could be partially offset by treatment with SNP. HS also significantly affected the $\mathrm{NAD}^{+} / \mathrm{NADH}$ ratio (HS vs. control, $P=3.429 \times 10^{-9}$ ), and the addition of exogenous SNP alleviated the imbalance in NAD caused by HS. In contrast, the addition of cPTIO significantly decreased the $\mathrm{NAD}^{+} / \mathrm{NADH}$ ratio compared with that observed in the HS group (cPTIO_HS vs. HS, $P=0.007$ ) (Fig. 1F). Because most $\mathrm{NAD}^{+}$is reduced to NADH by the TCA cycle in mitochondria and NADH serves as a substrate for the generation of ROS in the respiratory chain [37], it can be assumed that the TCA cycle is accelerated by $\mathrm{HS}$ and that NO can affect the accumulation of NADH by regulating the TCA cycle. ATP is commonly considered as intracellular energy currency molecule, and the mitochondrial respiratory chain is the site of ATP production. As shown in Fig. 1G, the total respiratory rate of mycelia decreased after HS, and this decrease might be caused by mitochondrial damage induced by HS. The addition of exogenous NO further inhibited the total respiratory rate. It has been suggested that $\mathrm{NO}$ can regulate the respiratoy rate of mycelia under stress and that NO might reduce ROS production by inhibiting the respiratory rate. Interestingly, the ATP content increased by $39.27 \%$ after HS treatment compared with the level found in the control group, and this increase was synchronous with the inceases in $\mathrm{O}_{2}{ }^{-}$and $\mathrm{H}_{2} \mathrm{O}_{2}$. The increase in the ATP content might be due to the observed increase in the NADH levels (Fig. 1E). Exogenous NO significantly inhibited the increase in ATP induced by $\mathrm{HS}\left(\mathrm{SNP}_{-} \mathrm{HS}\right.$ vs. HS, $P=2.237 \times 10^{-6}$ ), whereas the addition of exogenous CPTIO significantly increased the ATP content under HS (cPTIO_HS vs. HS, $P=0.001)($ Fig. $1 \mathrm{H})$. The results further suggested that NO can regulate the respiratory chain. 


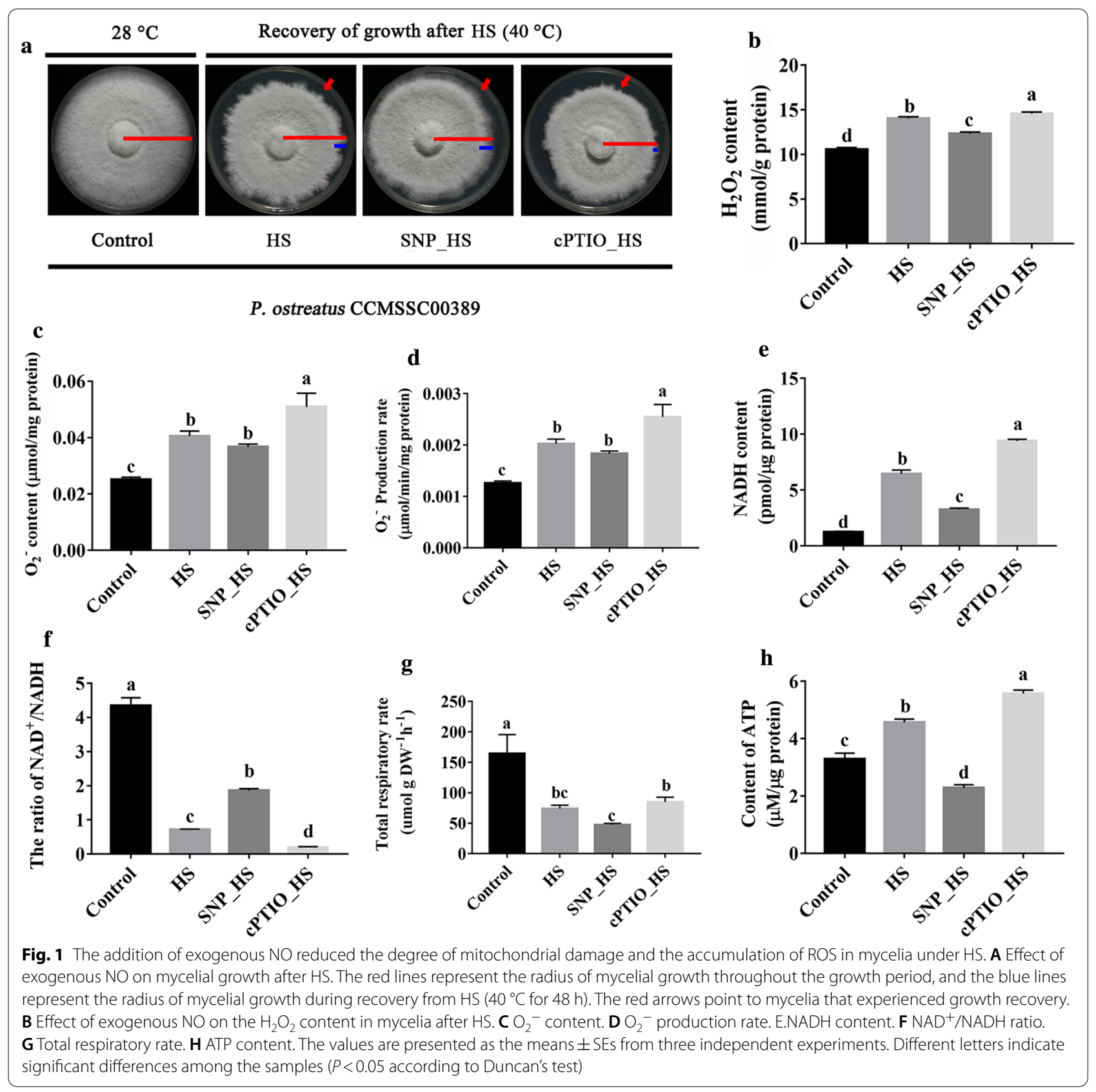

In conclusion, $\mathrm{NO}$ might alleviate the inceases in $\mathrm{O}_{2}{ }^{-}$ and $\mathrm{H}_{2} \mathrm{O}_{2}$ by regulating the mitochondrial respiratory chain and thereby reducing mycelial damage induced by HS.

\section{RNA-Seq analysis of the regulatory mechanism} through which NO alleviates mycelial damage induced by HS

To fully understand the effect of NO on the transcriptome of $P$. ostreatus under HS, 12 RNA-Seq cDNA libraries were prepared. As shown in Additional file 1: Table S2, after removing adapters, low-quality regions and all possible contamination, each treatment group contained an average of $47.29 \mathrm{M}$ clean reads with a quality score of 30 (Q30) $>94.93 \%$ and a GC percentage between 53.03 and $53.64 \%$. The ratio of reads mapping to the P. ostreatus genome was high, ranging from 77.26 to $82.93 \%$. This result indicated that the accuracy of the sequencing results was high and could be used for subsequent analysis. 
Based on the RNA-Seq analysis, the functions of 579 differentially expressed genes (DEGs) identified from the SNP_HS vs. cPTIO_HS comparison were examined to elucidate the possible mechanism through which $\mathrm{NO}$ alleviates the oxidative damage to mycelia induced by HS (Fig. 2). In the gene ontology (GO) analysis, the DEGs identified from the SNP_HS vs. cPTIO_HS comparison were classified into three categories: 'biological process', 'cellular component' and 'molecular function' (Fig. 3). Within the biological process category, genes corresponding to metabolic processes, cellular processes and single organism process were the most abundant. Within the cellular component category, the most abundant terms of DEGs were membrane and membrane parts, and within the molecular function category, catalytic activity and binding were the most abundant terms. These results indicated that NO might participate in the mycelial response to HS by regulating cell metabolism, affecting cell membrane components and structure, and affecting the catalytic activity of proteins.

To understand the functions of the DEGs identified from the SNP_HS vs. cPTIO_HS comparison, a pathway enrichment analysis was performed. The results showed that the DEGs were mainly concentrated in the following pathways: oxidoreductase activity, oxidation-reduction process, cofactor binding, protein kinase activity, phosphotransferase activity, alcohol group as acceptor, and protein phosphorylation (Fig. 4A). Previous studies have shown that HS can lead to the production and accumulation of ROS in mycelia, and ROS can further cause oxidative damage. As shown in Fig. 4A, exogenous $\mathrm{NO}$ can affect the oxidation-reduction process and oxidoreductase activity. In addition, considering the close relationship between the antioxidant system and ROS clearance, the expression pattern of the genes after the addition of an $\mathrm{NO}$ donor or scavenger was further analyzed with a heatmap. As shown in Fig. 4B, 69 DEGs identified after the addition of SNP or CPTIO were enriched in the oxidation-reduction process pathway and oxidoreductase activity, and these included 62 significantly upregulated DEGs and seven downregulated DEGs. It can thus be hypothesized that NO can activate the activity of oxidoreductase and accelerate redox reactions under HS.

\section{NO affected the expression of key genes in the respiratory chain under $\mathrm{HS}$}

The respiratory chain is closely related to ROS. To further explore whether $\mathrm{NO}$ can regulate the respiratory chain to alleviate mycelial damage under HS, six DEGs related to the respiratory chain were identified from 579 DEGs via functional enrichment (Table 1). Two of these DEGs were not annotated, and the remaining four DEGs were g3097, g11376, g12148 and g12952, which encode the succinate dehydrogenase iron-sulfur subunit, AOX, hypothetical protein and the mitochondrial chaperone BCS1, respectively. As illustrated in the heatmap shown in Fig. 5, an exogenous NO donor (SNP) inhibited the expression of five of the DEGs and upregulated the expression of g11376 (AOX). The mitochondrial chaperone BCS1 is a transmembrane chaperone found in the mitochondrial inner membrane and it is required for the assembly of mitochondrial respiratory chain complex III (http://www. ebi.ac.uk/interpro/entry/InterPro/IPR027243/) [38]. The

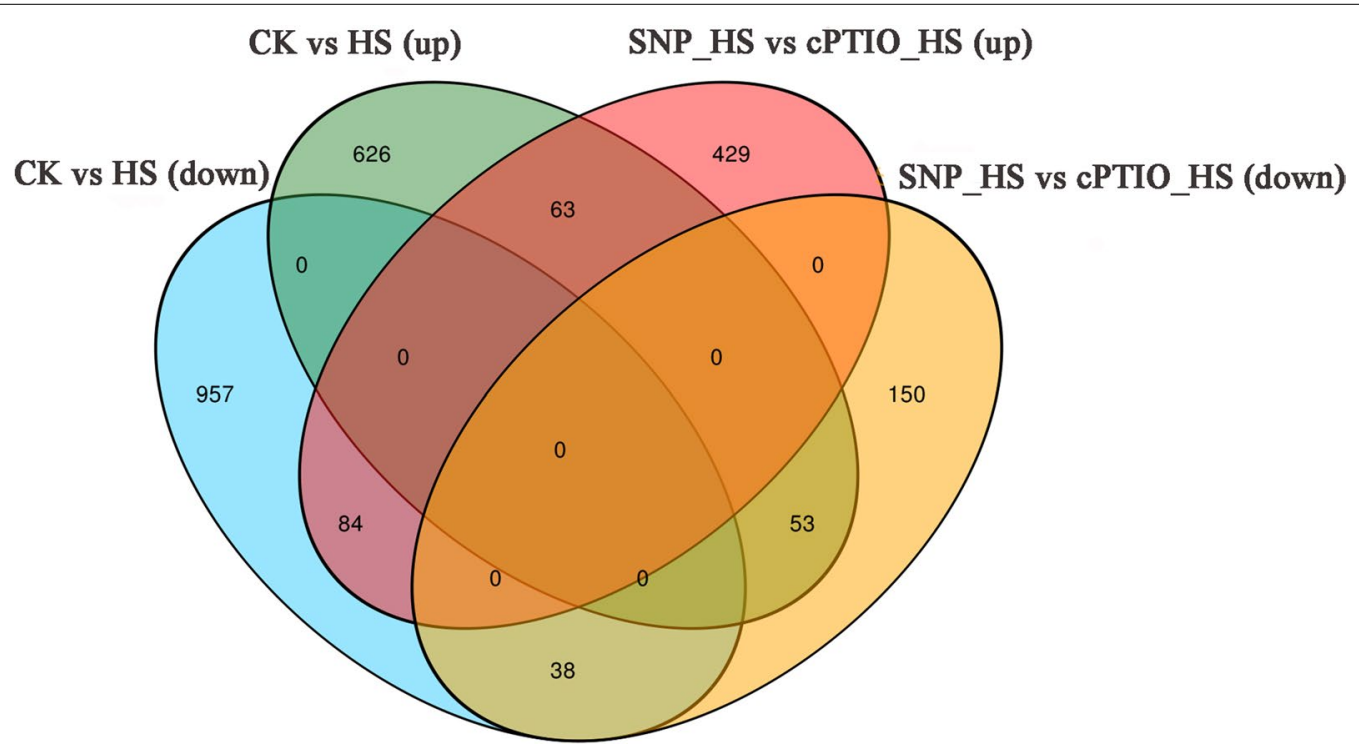

Fig. 2 Number of up and downregulated genes among the CK, HS, SNP_HS and CPTIO_HS groups 


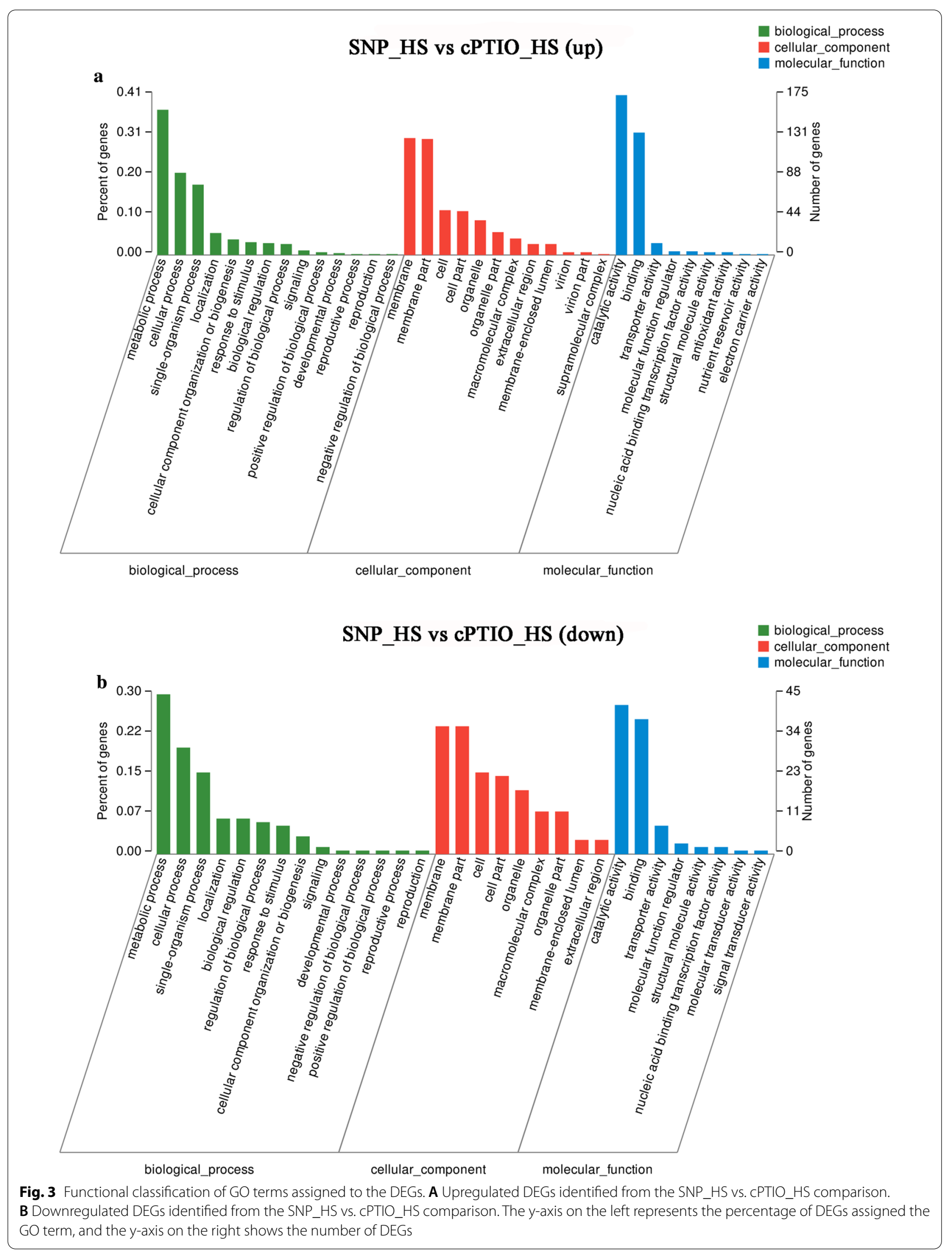


succinate dehydrogenase iron-sulfur subunit is involved in the synthesis and assembly of mitochondrial respiratory chain complex II (https://www.uniprot.org/uniprot/ A1AZJ0). These results indicate that exogenous $\mathrm{NO}$ can inhibit the cytochrome pathway and activate the alternative oxidation pathway.

\section{NO induced aox gene expression under HS}

ROS are mainly produced by the respiratory chain under high oxygen conditions and in a high reduction state during the transition of mitochondria from complex III to complex IV, which results in the leakage of a large number of electrons and the reduction of oxygen molecules. AOX prevents excessive reduction of downstream complexes (cytochrome pathway) by introducing a branch into the ETC at the ubiquinone pool. When AOX bypasses complexes III and IV of the cytochrome pathway, it significantly reduces ATP production and single electron leakage, which results in the reduction of ROS production [29]. Moreover, as determined through RNA-Seq analysis, aox can be regulated by NO, participates in the oxidation-reduction process pathway and has oxidoreductase activity.

To further verify the regulatory effect of $\mathrm{NO}$ on $a o x$ under HS, the effects of exogenous NO donors and scavengers on aox gene expression were assessed. As shown in Fig. 6A, the relative expression of the aox gene in $P$. ostreatus mycelia changed steadily with increases in the time of exposure to HS. During the first $24 \mathrm{~h}$ of exposure to HS, aox gene expression first increased and then decreased within a small range. An increase in the exposure time to $48 \mathrm{~h}$ significantly increased the relative expression of the aox gene, and the level detected after $48 \mathrm{~h}$ was approximately 8 -fold higher than that at $0 \mathrm{~h}$. As shown in Fig. 6B, the relative expression of the aox gene was significantly increased after HS (HS vs. control, $P=6.195 \times 10^{-5}$ ), whereas exogenous SNP treatment almost completely enhanced this effect (SNP vs. HS, $P=0.015)$, and cPTIO blocked the effect of SNP on aox gene expression (cPTIO vs. SNP, $P=5.704 \times 10^{-6}$ ). The results showed that $\mathrm{NO}$ can promote the expression of the aox gene in mycelia after HS. In conclusion, NO might participate in the response of $P$. ostreatus to HS by regulating the expression of the aox gene.

\section{The OE of aox promoted the recovery of mycelial growth after HS}

Using previously reported methods [39], we successfully constructed an RNAi-aox plasmid (Additional file 1: Fig. S1) and established aox-transformed strains via Agrobacterium-mediated transformation. The hyg gene fragment

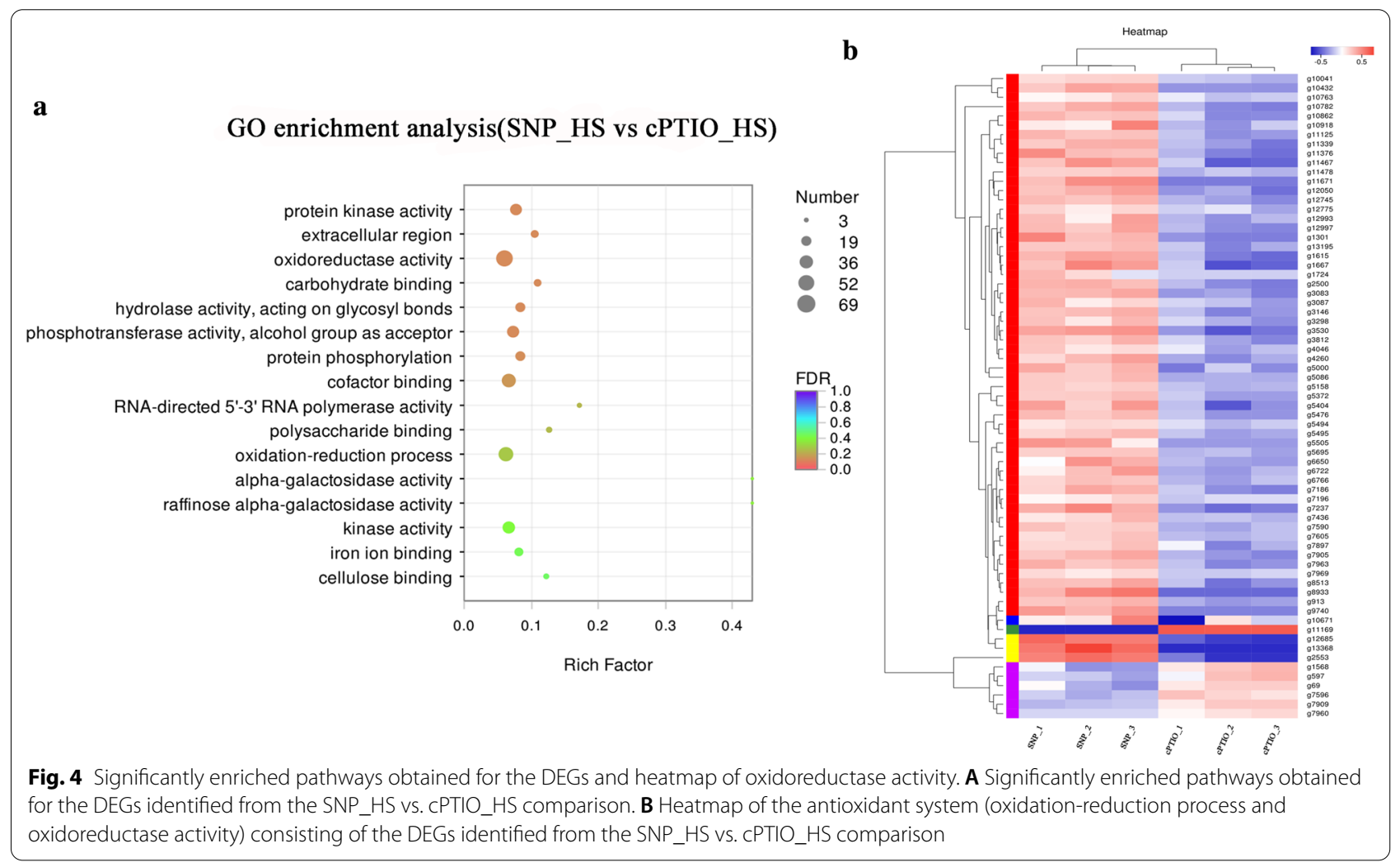




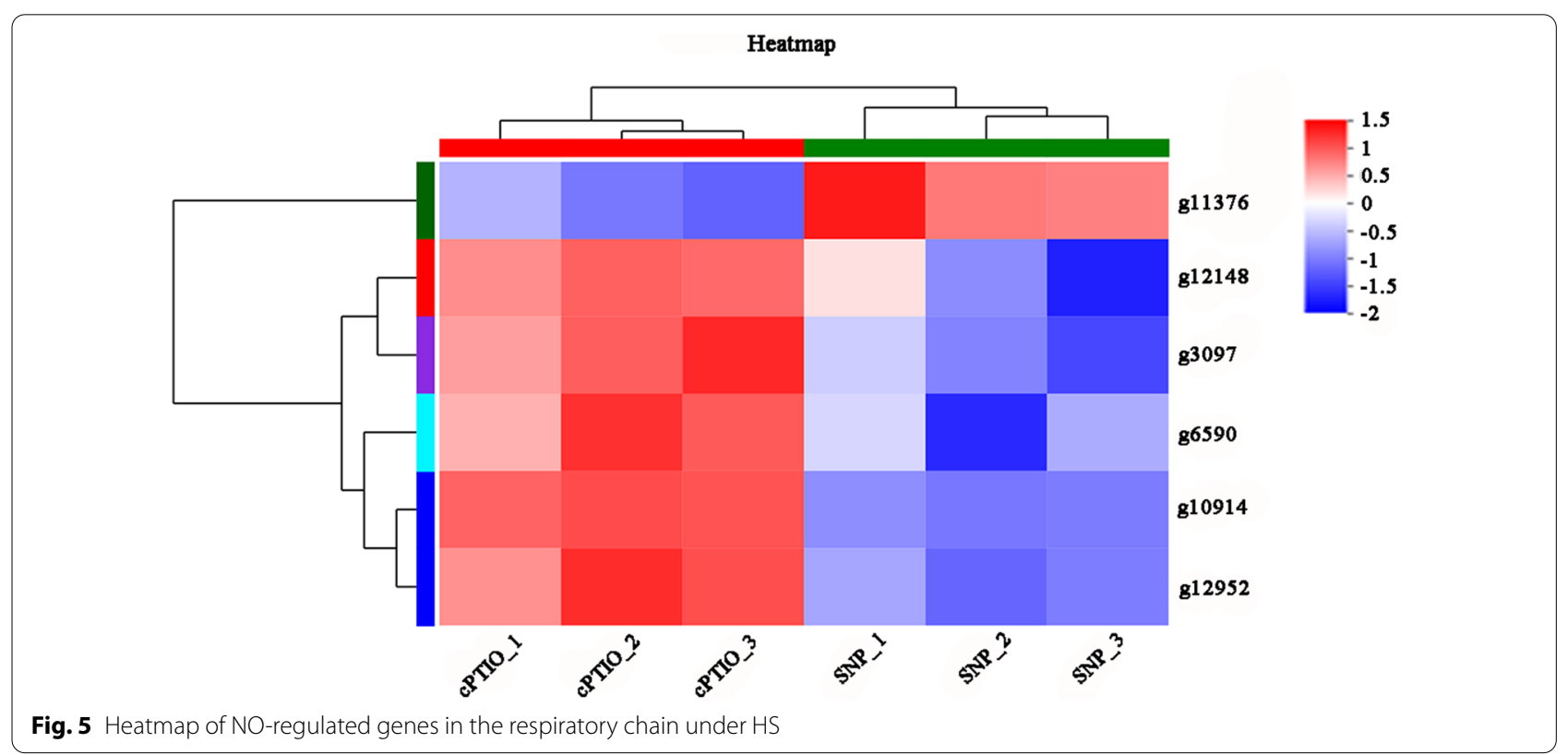

Table 1 List of genes related to the respiration that are regulated by $\mathrm{NO}$

\begin{tabular}{ll}
\hline Gene ID & Gene description \\
\hline g3097 & Succinate dehydrogenase iron-sulfur subunit \\
g6590 & - \\
g11376 & Alternative oxidase \\
g12148 & Hypothetical protein PLEOSDRAFT_1096427 \\
g12952 & (P. ostreatus PC15) \\
g10914 & Mitochondrial chaperone BCS1 \\
\hline
\end{tabular}

was amplified for preliminary selection, and the relative expression of this target gene was amplified by quantitative reverse transcription PCR (RT-qPCR) to screen the RNAi-aox strains. The results are shown in Additional file 1: Fig. S2. To further explore the function of the $a o x$ gene in the response of $P$. ostreatus to HS, plates containing the various strains were cultured at $28{ }^{\circ} \mathrm{C}$ for $5 \mathrm{~d}$, transferred to $40{ }^{\circ} \mathrm{C}$ for $48 \mathrm{~h}$, and then incubated at $28^{\circ} \mathrm{C}$ to allow growth recovery. After $3 \mathrm{~d}$ of growth recovery after HS, mycelial germination was observed in the wild type (WT), OE-aox and RNAi-aox strains, as shown in Fig. 7A. After $5 \mathrm{~d}$ of growth recovery, compared with the WT strain, the OE-aox strains exhibited a faster recovery rate and a complete colony edge, whereas RNAi-aox strains presented a slower mycelial recovery rate and showed defects on the edge of the colony. In conclusion, the aox gene plays an active role in the recovery of P. ostreatus mycelia after HS. Figure $7 \mathrm{~B}, \mathrm{C}$ and $\mathrm{D}$ show the changes in the $\mathrm{H}_{2} \mathrm{O}_{2}$ content, the $\mathrm{O}_{2}{ }^{-}$content and the production rate of the $a o x$-transformed strains under HS. Under HS, the accumulation of $\mathrm{H}_{2} \mathrm{O}_{2}$ in the OE-aox 47 and OE-aox 71 strains was significantly lower (by 9.05 and $12.28 \%$, respectively) than that in the WT strain. In addition, the $\mathrm{H}_{2} \mathrm{O}_{2}$ content in the $\mathrm{OE}$-aox 34 strain was $4.29 \%$ lower than that in the WT strain. In contrast, the $\mathrm{H}_{2} \mathrm{O}_{2}$ contents in the RNAi-aox 12, RNAi-aox 29 and RNAi-aox 7 strains were $4.59 \%, 17.71$ and $21.11 \%$ higher, respectively, than that in the WT strain. As shown in Figs. $7 \mathrm{C}$ and $\mathrm{D}$, the $\mathrm{O}_{2}{ }^{-}$production rate and content of the OE-aox strains under HS were significantly lower than those of the WT strain, whereas those of the RNAi -aox strains increased significantly. These results indicated that the aox gene can regulate the production and accumulation of ROS. ROS are mainly caused by electron leakage in the respiratory chain. As shown in Figs. 7E and $\mathrm{G}$, compared with the WT strain, the average NADH and ATP contents in the OE-aox strains were decreased by 26.47 and $9.82 \%$, respectively, and the average $\mathrm{NAD}^{+}$/ $\mathrm{NADH}$ ratio in these strains was 1.55 -fold higher. In contrast, the average NADH and ATP contents of the RNAiaox strains were 66.86 and $29.79 \%$ higher, respectively, than those in the WT strain, and the $\mathrm{NAD}^{+} / \mathrm{NADH}$ ratio was decreased by $26.52 \%$. Therefore, it can be speculated that the aox gene plays an important role in regulating the mitochondrial ETC and energy metabolism and in maintaining mitochondrial homeostasis.

In conclusion, the $a o x$ gene can affect the production and accumulation of ROS by regulating mitochondrial respiration, and it participates in the mycelial response to HS. 


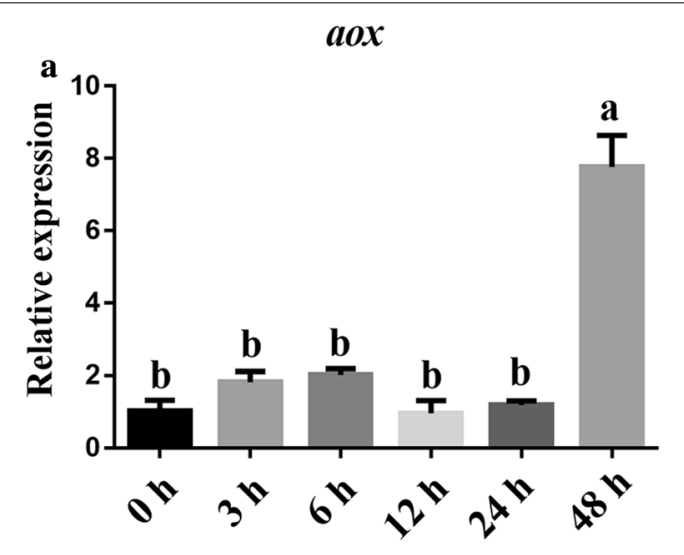

Heat treatment time

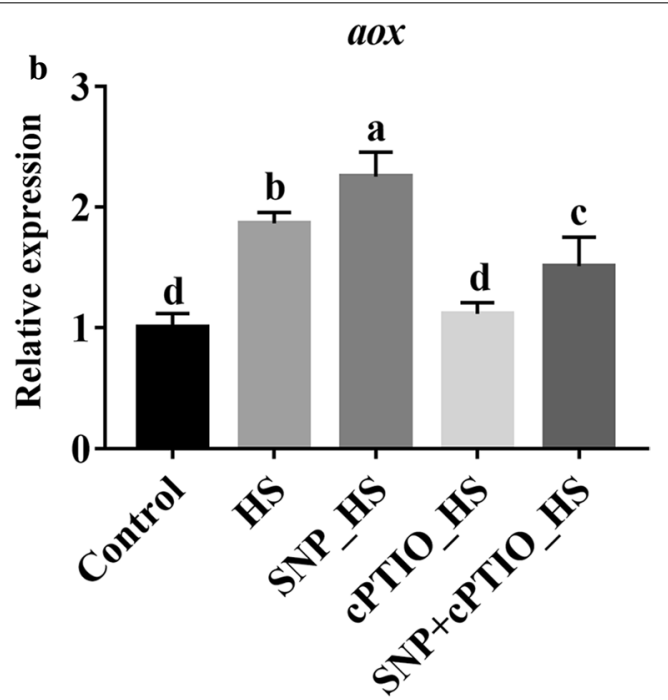

Fig. $6 \mathrm{NO}$ induced aox gene expression in P. ostreatus under HS. A Expression of the aox gene after exposure to HS for different amounts of time. B Effect of $\mathrm{NO}$ on the expression of the aox gene under HS. The values are presented as the means \pm SEs from three independent experiments. Different letters indicate significant differences among the samples ( $P<0.05$ according to Duncan's test)

\section{The aox gene regulates the expression of key antioxidant enzyme genes in mycelia after HS}

The signaling from organelles that controls nuclear gene expression is called retrograde signaling, and previous studies have shown that aox serves as a marker gene for mitochondrial retrograde regulation [40]. AOX also acts as a facilitator for signaling molecules conveying the metabolic status of mitochondria to the nucleus and is thus able to influence nuclear gene expression [41]. Moreover, studies have shown that AOX can affect the production and accumulation of ROS. Antioxidant systems (such as antioxidant enzymes and nonenzymatic oxidants) play critical roles in the defense against oxidative stress [42]. We measured the changes in expression of genes that encode four key antioxidant enzymes, namely, catalase (CAT), superoxide dismutase (SOD), thioredoxin reductase (TrxR) and glutathione peroxidase (GSH-PX). In the genome of $P$. ostreatus, two genes (cat1 and cat 2 ) encode CAT, four genes ( $\operatorname{sod} 1, \operatorname{sod} 2$, $\operatorname{sod} 3$ and $\operatorname{sod} 4)$ encode SOD, one gene encodes TrxR, and one gene encodes GSH-PX [43]. To study whether aox in P. ostreatus can alleviate ROS stress by regulating the antioxidant enzyme system, we measured the expression of these key antioxidant enzyme genes in aox-transformed strains exposed to $\mathrm{HS}$ at $40{ }^{\circ} \mathrm{C}$ for $48 \mathrm{~h}$. As shown in Fig. $8 \mathrm{~A}$, after $48 \mathrm{~h}$ of exposure to HS, the relative expression of cat1 in the OE-aox 47-, OE-aox 71- and OE-aox 34-transformed strains was significantly downregulated to $26.5 \%, 25.96$ and $35.30 \%$ respectively, of the level found in the WT strain. In the RNAi-aox 12 and RNAi-aox 29 strains, the relative expression of cat 1 was significantly increased by
1.57 -fold and 7.28-fold, respectively, compared with that found in the WT strain, but no significant change in the expression of this gene was detected in the RNAi-aox 7 strain. As shown in Fig. 8B, compared with that in the WT strain, the relative expression of cat 2 in the OE$a o x$ and RNAi-aox strains was significantly downregulated and upregulated, respectively. In conclusion, $a o x$ can negatively regulate cat gene expression under HS. As shown in Figs. $8 \mathrm{C}$ and $\mathrm{D}$, the expression of the trxr and $g s h-p x$ genes was significantly downregulated in the OE-aox-transformed strains. In addition, the trxr gene was significantly upregulated in all RNAi-aox strains, and the relative expression of the $g s h-p x$ gene was significantly upregulated in RNAi-aox12 and RNAi-aox 29 and slightly upregulated in RNAi-aox 7. SOD is one of the major defense systems used to remove $\mathrm{O}_{2}{ }^{-}$. The relative expression levels of the four SOD-encoding genes in the aox-transformed strains under HS are shown in Figs. 8E-H. As shown in Fig. 8E, compared with the level in the WT strain, sod1 gene expression was significantly increased in the OE-aox strains and decreased to $72.66 \%, 67.71$ and $79.93 \%$ in RNAi-aox 12, RNAi-aox 29 and RNAi-aox 7, respectively. In addition, as shown in Figs. 8F-H, the expression levels of $\operatorname{sod} 2$, sod 3 and $\operatorname{sod} 4$ in the OE-aox strains were significantly downregulated, to $34.93 \%, 60.33$ and $32.16 \%$, respectively, of the level found in the WT strain. However, sod2 and sod 3 gene expression was not significantly changed in the RNAiaox strains, and the expression of sod4 was slightly downregulated in these strains compared with the WT strain. Because the sod 1 gene encodes Gu-SOD, which mainly 


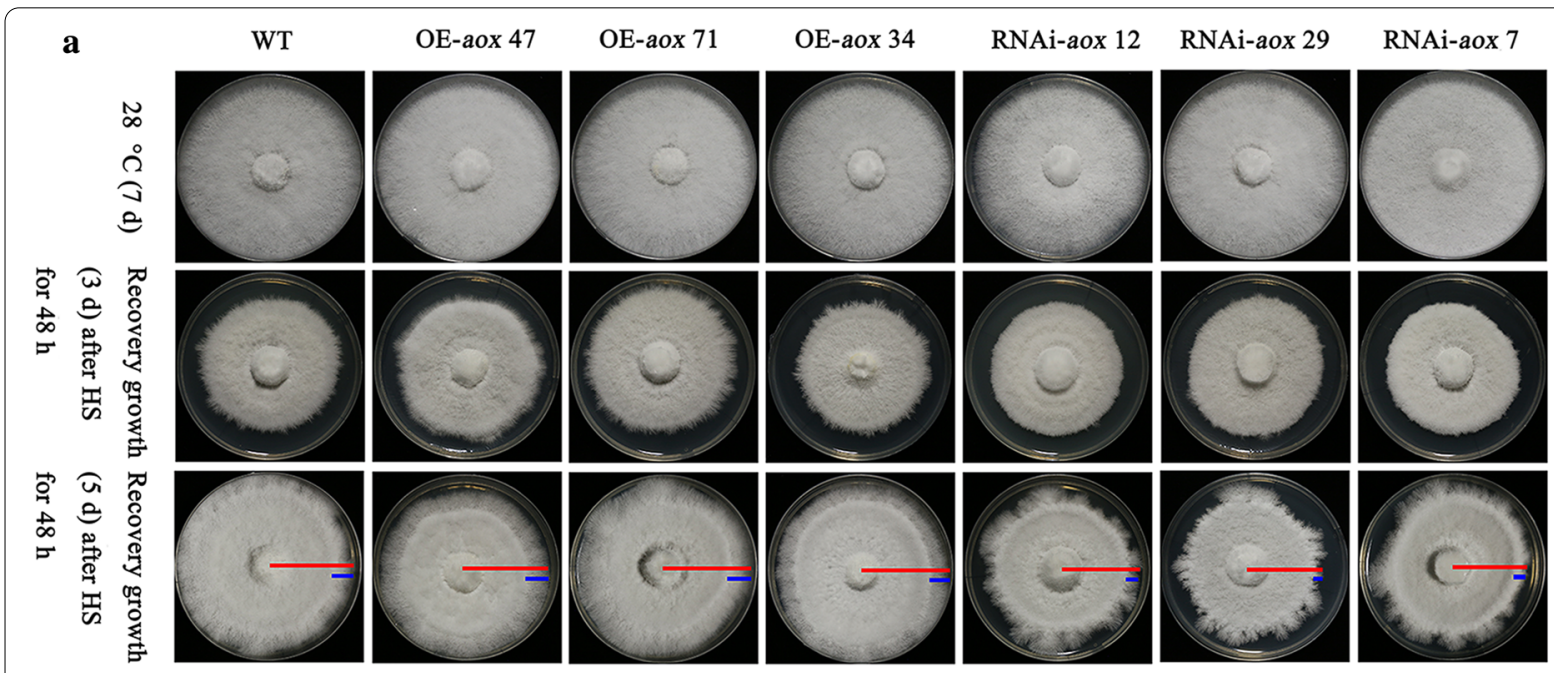

b

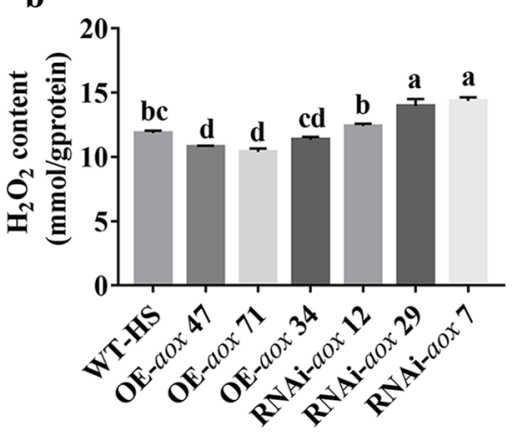

e

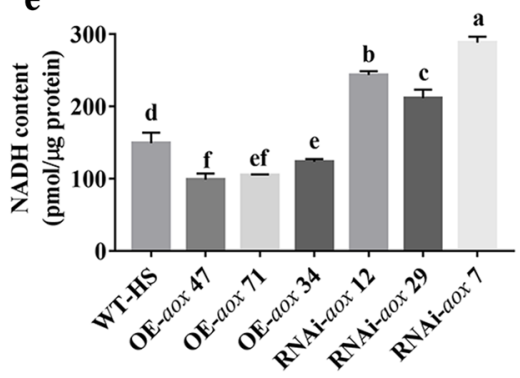

c

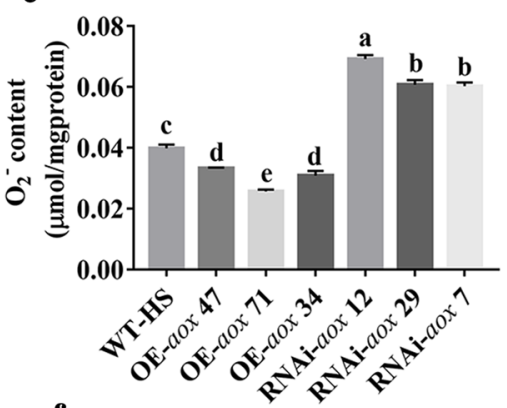

f

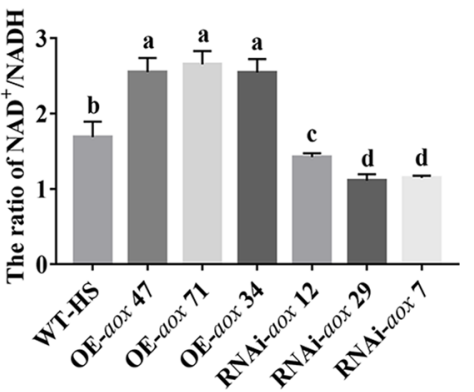

d

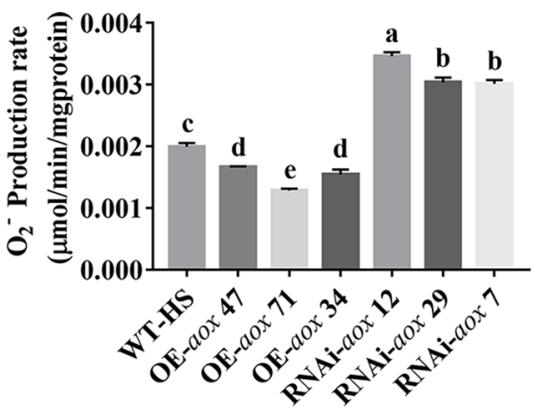

$\mathbf{g}$

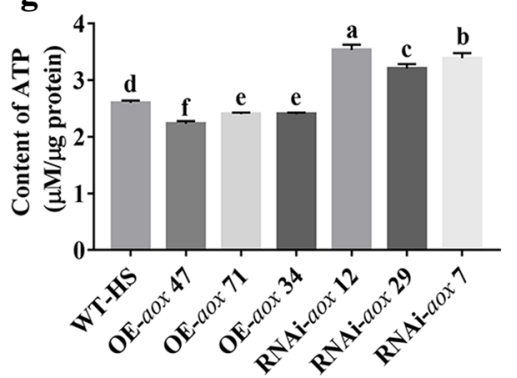

Fig. 7 aox affects ROS production and promotes mycelial growth by regulating the respiratory pathway under HS. A Recovery of WT, OE-aox and RNAi-aox strains after HS. The red lines represent the radius of mycelial growth throughout the growth period, and blue lines represent the radius of mycelial growth during the $48 \mathrm{~h}$ of recovery after $\mathrm{HS}$. $\mathbf{B ~} \mathrm{H}_{2} \mathrm{O}_{2}$ content. $\mathrm{C}_{2}{ }^{-}$content. $\mathbf{D} \mathrm{O}_{2}{ }^{-}$production rate. E NADH content. F NAD ${ }^{+} / \mathrm{NADH}$ ratio. G ATP content. The values are presented as the means \pm SEs from three independent experiments. Different letters indicate significant differences among the samples $(P<0.05$ according to Duncan's test)

exists in the cytoplasm, it can be concluded that the $\mathrm{OE}$ of aox during $\mathrm{HS}$ can regulate the expression of SODencoding genes.

In conclusion, in addition to SOD1, the OE-aox strains exhibited downregulated expression of key antioxidant enzyme coding genes under $\mathrm{HS}\left(40^{\circ} \mathrm{C}\right.$ for $\left.48 \mathrm{~h}\right)$, and the
RNAi-aox strains exhibited upregulated expression of CAT-, GSH-PX- and TrxR-encoding genes. It can thus be hypothesized that the significant upregulation of the aox gene after $\mathrm{HS}\left(40{ }^{\circ} \mathrm{C}\right.$ for $\left.48 \mathrm{~h}\right)$ can further affect the expression of key antioxidant enzyme-encoding genes. 


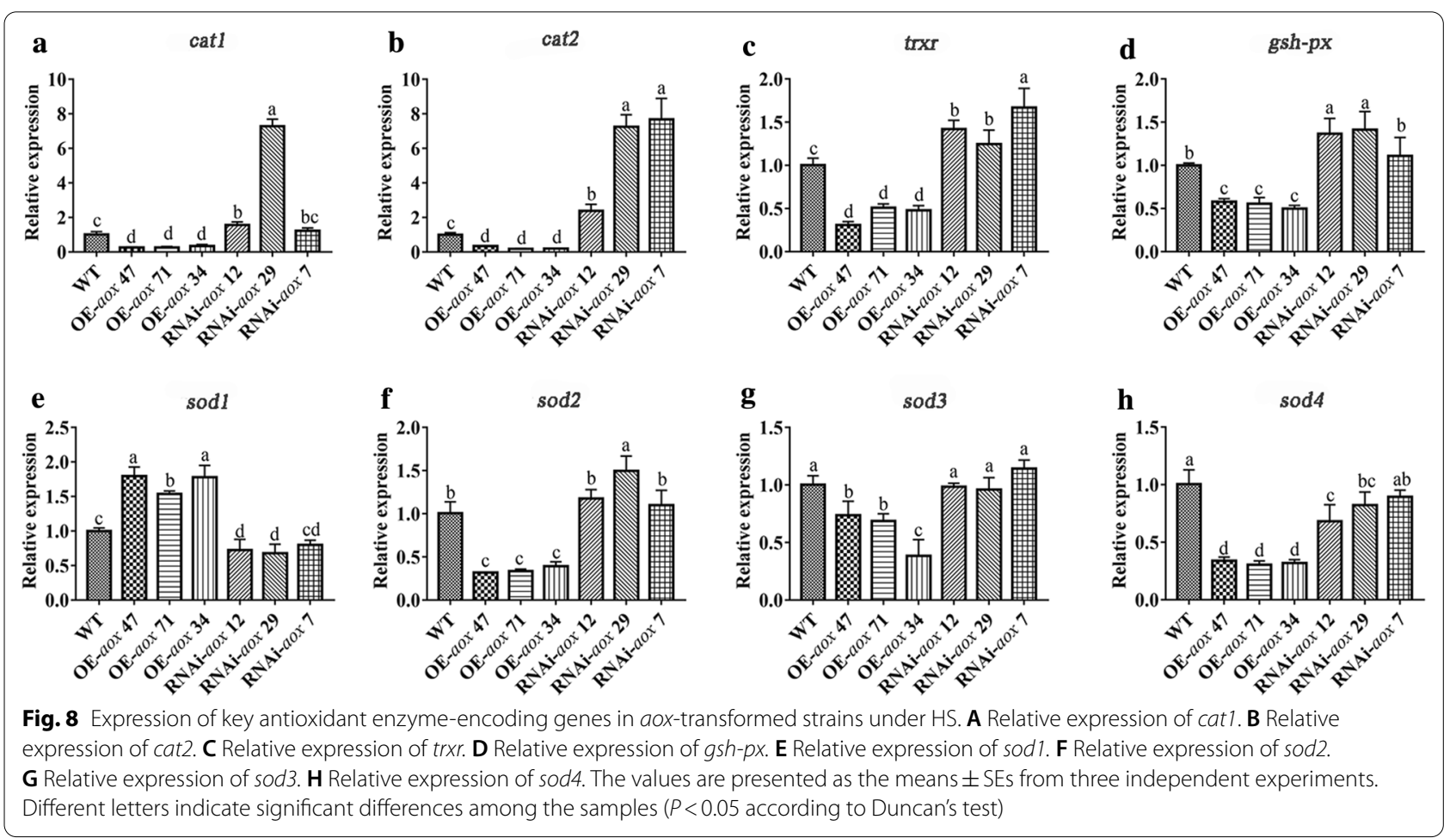

\section{Exogenous benzohydroxamate (BHAM) regulated the expression of antioxidant enzyme-encoding genes in mycelia under $\mathrm{HS}$}

AOX is insensitive to inhibitors of the cytochrome pathway such as cyanide, antimycin $\mathrm{A}$, and miyxothiazol, but is inhibited by primary hydroxamic acids, such as BHAM [44, 45]. To further prove whether aox interference can regulate the expression of key antioxidant enzyme-encoding genes, an experiment in which different concentrations of an AOX inhibitor (BHAM) were added was then performed. The results showed that the growth rate of $P$. ostreatus mycelia at $28{ }^{\circ} \mathrm{C}$ was affected by exogenous BHAM. As shown in 1: Figs. S3A, B, a low concentration of BHAM (50-100 $\mu \mathrm{M})$ had no significant effect on the colony and mycelial growth rate of $P$. ostreatus, but the addition of exogenous BHAM at a concentration higher than $200 \mu \mathrm{M}$ affected overall mycelial growth and significantly reduced the mycelial growth rate. Furthermore, whether exogenous BHAM could regulate the expression of the aox gene was assessed in the experiment. As shown in Additional file 1: Fig. S3C, the addition of BHAM at a concentration of 50-200 $\mu \mathrm{M}$ significantly downregulated the expression of the aox gene compared with the control (CK) level, which indicated that low concentrations of BHAM can inhibit the expression of the aox gene. However, a BHAM concentration of $400 \mu \mathrm{M}$ upregulated the expression of the aox gene, and because this concentration also significantly inhibited the mycelial growth rate, it can be speculated that $400 \mu \mathrm{M}$ BHAM might affect the growth environment of mycelia and induce abiotic stress, which eventually leads to the induction of aox gene expression. In addition, because the mycelial growth of the RNAi-aox strains did not significantly differ from the normal growth of $P$. ostreatus mycelia, 50-200 $\mu \mathrm{M}$ was used as the BHAM concentration in the subsequent experiments.

As shown in Fig. 9A, the addition of exogenous BHAM at different concentrations slowed the recovery of mycelial growth after HS compared with that of the WT strain, and this result was consistent with the results obtained with the RNAi-aox strains. The effects of exogenous BHAM on the $\mathrm{H}_{2} \mathrm{O}_{2}$ content and the $\mathrm{O}_{2}{ }^{-}$content and production rate were then assessed. The results showed that compared with the CK-HS group, the $\mathrm{H}_{2} \mathrm{O}_{2}$ content and $\mathrm{O}_{2}{ }^{-}$content and production rate of mycelia under HS were significantly increased by different concentrations of BHAM (Figs. 9B and D). These results indicated that exogenous BHAM could promote the production and accumulation of ROS in mycelia under HS. The effects of exogenous BHAM on the expression of key antioxidant enzyme-encoding genes in mycelia under HS are shown in Figs. 9E-L. As illustrated in the figures, the addition of exogenous BHAM significantly increased the expression of antioxidant enzyme-encoding genes under HS. Specifically, the expression levels of cat1 (Fig. 9E), cat2 (Fig. 9F), trxr (Fig. 9G), gsh-px (Fig. 9H), sod2 
(Fig. 9J) and sod4 (Fig. 9L) increased with increases in the BHAM concentration, and the highest expression levels of these genes, which were 47.25-fold, 17.76-fold, 1.99fold, 6.86-fold, 4.76-fold and 3.98-fold higher than those found in the HS group, respectively, were detected with a BHAM concentration of $200 \mu \mathrm{M}$. In addition, the highest expression levels of $\operatorname{sod} 1$ and $\operatorname{sod} 3$, which were 1.90-fold and 2.42-fold higher than the control levels, respectively, were obtained with the exogenous addition of $100 \mu \mathrm{M}$ BHAM (Figs. 9I and K).

In conclusion, exogenous BHAM can promote the production and accumulation of ROS under HS and then regulate the expression of antioxidant enzyme-encoding genes.

\section{Discussion}

$\mathrm{NO}$ is a type of free radical involved in many types of stress and physiological processes and serves as a key regulator of physiological processes [46]. In this study, we revealed that NO reduces the production of ATP and ROS by activating the alternative oxidation pathway, maintaining mitochondrial function, and enhancing the resistance of $P$. ostreatus to $\mathrm{HS}$. In addition, AOX can act on the retrograde signaling pathway to regulate the expression of nuclear genes (antioxidant enzyme-encoding genes) in response to HS. This finding provides a new perspective on the fungal regulatory mechanism of $\mathrm{NO}$ in response to abiotic stress.

The regulatory effect of $\mathrm{NO}$ on metabolism during stress relief is not unusual. Recently, an increasing number of studies have shown that the application of exogenous NO is useful for allaying oxidative stresses caused by drought, high temperature and salinity [47-49]. HS refers to an increase in temperature to a level that exceeds the optimal growth temperature of the organism of interest, which causes irreversible damage to its growth [50]. HS induces an increased generation of ROS. The production rate of ROS is strongly dependent on the membrane potential, and a change in the membrane potential increases the probability of electron leakage and thus the production of $\mathrm{O}_{2}{ }^{-}$, which can be used as a substrate to produce $\mathrm{H}_{2} \mathrm{O}_{2}$ and hydroxyl radicals [51]. In addition, excessive production and accumulation of ROS can cause lipid peroxidation, membrane leakage, enzyme inactivation, and DNA fragmentation or mutation, which can result in serious cell damage [52, 53]. During its cultivation, $P$. ostreatus is often exposed to high temperature.
The results obtained in this study show that exogenous $\mathrm{NO}$ can reduce the content of NADH and the production of ATP under HS. Previous studies have shown that $\mathrm{NO}$ can regulate the $\mathrm{NAD}^{+} / \mathrm{NADH}$ ratio in Ganoderma lucidum under HS [54], which is consistent with our results. Second, the $\mathrm{NAD}^{+} / \mathrm{NADH}$ ratio is mainly regulated by the TCA cycle, and NADH serves as a substrate for the generation of ATP in the respiratory chain [37]. Therefore, it can be speculated that exogenous NO can affect ATP production by regulating the NADH content. In addition, the production and accumulation of ROS in mycelia was decreased by exogenous NO addition. Previous studies have shown that NO can reduce ROS accumulation under $\mathrm{HS}$ by regulating different pathways. For instance, in wheat, the addition of exogenous NO donors can activate antioxidant enzymes, which results in the reduction of ROS and improvements in the heat resistance of wheat coleoptiles [55]. In rice, NO might protect photosynthesis from $\mathrm{HS}$ and alleviate oxidative stress by scavenging ROS [56]. In yeast, NO may be involved in various stress response systems, including $\mathrm{H}_{2} \mathrm{O}_{2}$-induced apoptosis [57]. For example, in Saccharomyces cerevisiae, the activation of Mac1 transcription factors by NO produced under a high temperature-stress conditions is important for the activation of the copperdependent SOD1 [58]. The results of this study are consistent with those reported in previous studies. Because ROS are mainly caused by electron leakage in the respiratory chain, it can be speculated that exogenous NO can reduce the production and release of ROS under HS by regulating the respiratory chain.

Stress-induced gene expression changes are key components of the molecular mechanism underlying plant adaptation to environmental challenges [59]. In recent years, RNA-Seq has become an effective means to study changes in gene expression under stress. For example, in Camellia sinensis, a total of 89 putative AP2/ERF transcription factors related to the temperature response were identified by RNA-Seq [60]. Under drought stress, 5689 DEGs in Populus trichocarpa leaves were obtained by RNA-Seq analysis, including genes related to the drought stress response and involved in photosynthesis, cell wall organization, and osmoprotectant metabolism [61]. Therefore, RNASeq is an effective method for exploring the stress response mechanisms of organisms based on changes in gene expression. In this study, RNA-Seq was used

(See figure on next page.)

Fig. 9 Exogenous BHAM regulates the expression of antioxidant enzymes under HS. A Exogenous BHAM inhibited the growth of mycelial growth

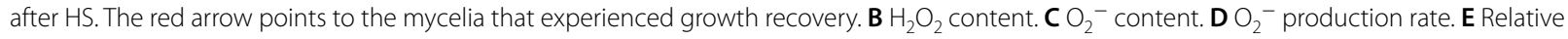
expression of cat1. F Relative expression of cat2. G Relative expression of trxr. $\mathbf{H}$ Relative expression of gsh-px. I Relative expression of sod1.

$\mathbf{J}$ Relative expression of sod2. K Relative expression of sod3. L Relative expression of sod4. The values are presented as the means \pm SEs from three independent experiments. Different letters indicate significant differences among samples ( $P<0.05$ according to Duncan's test) 

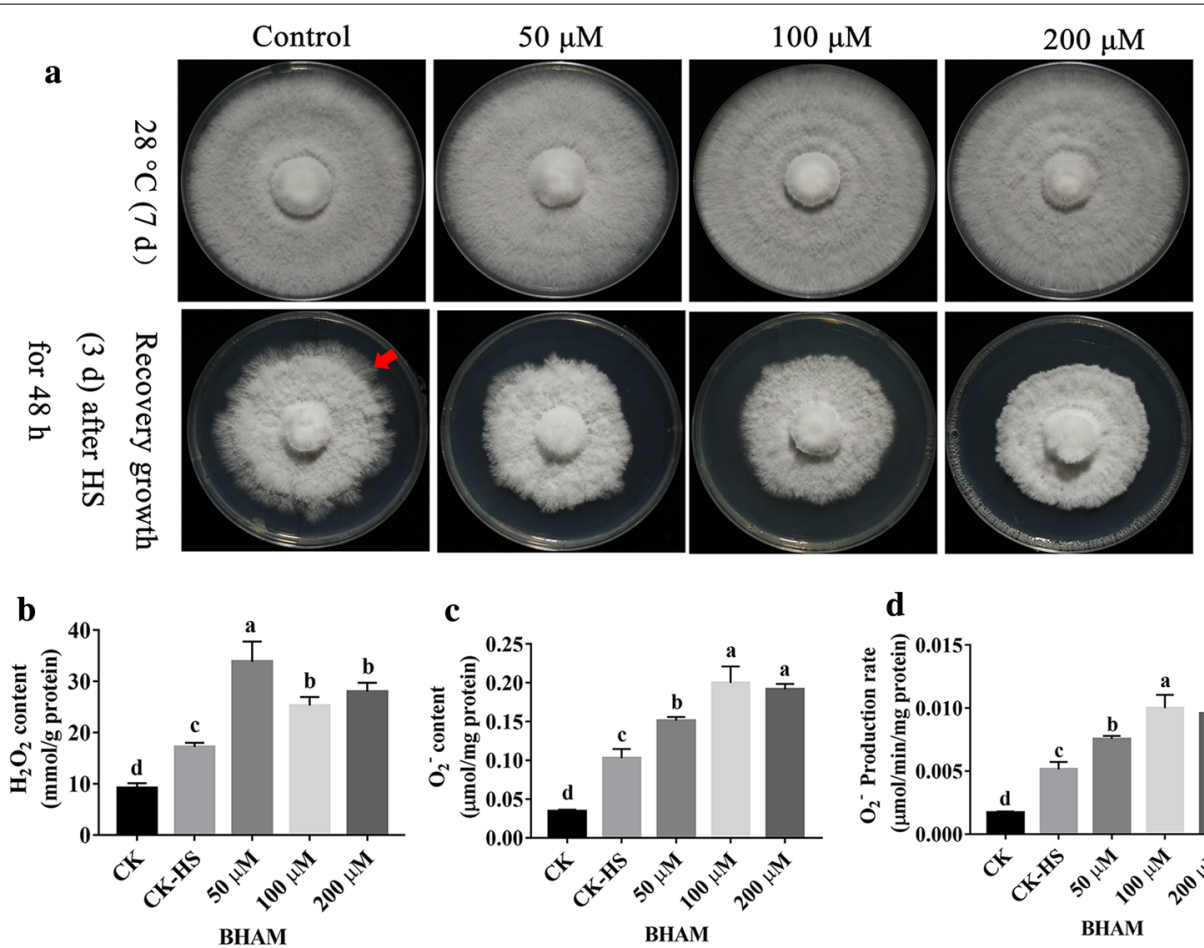

c

\section{d}
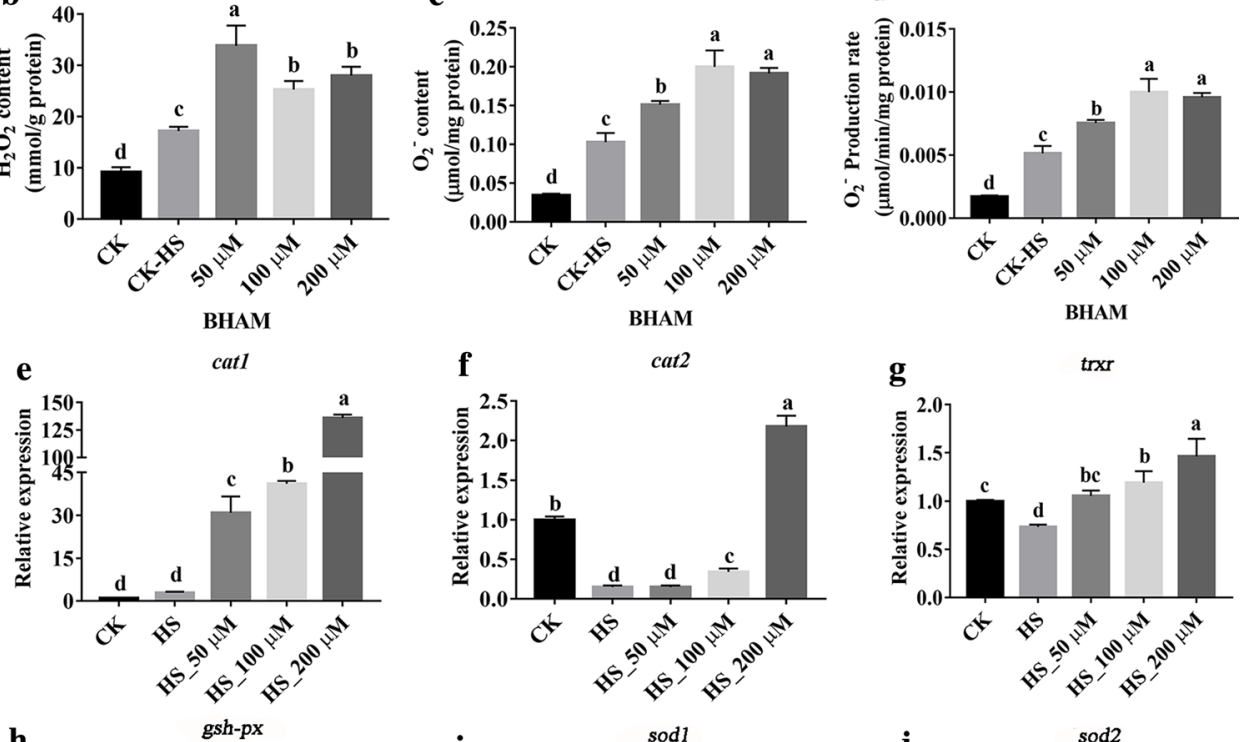

g trxr
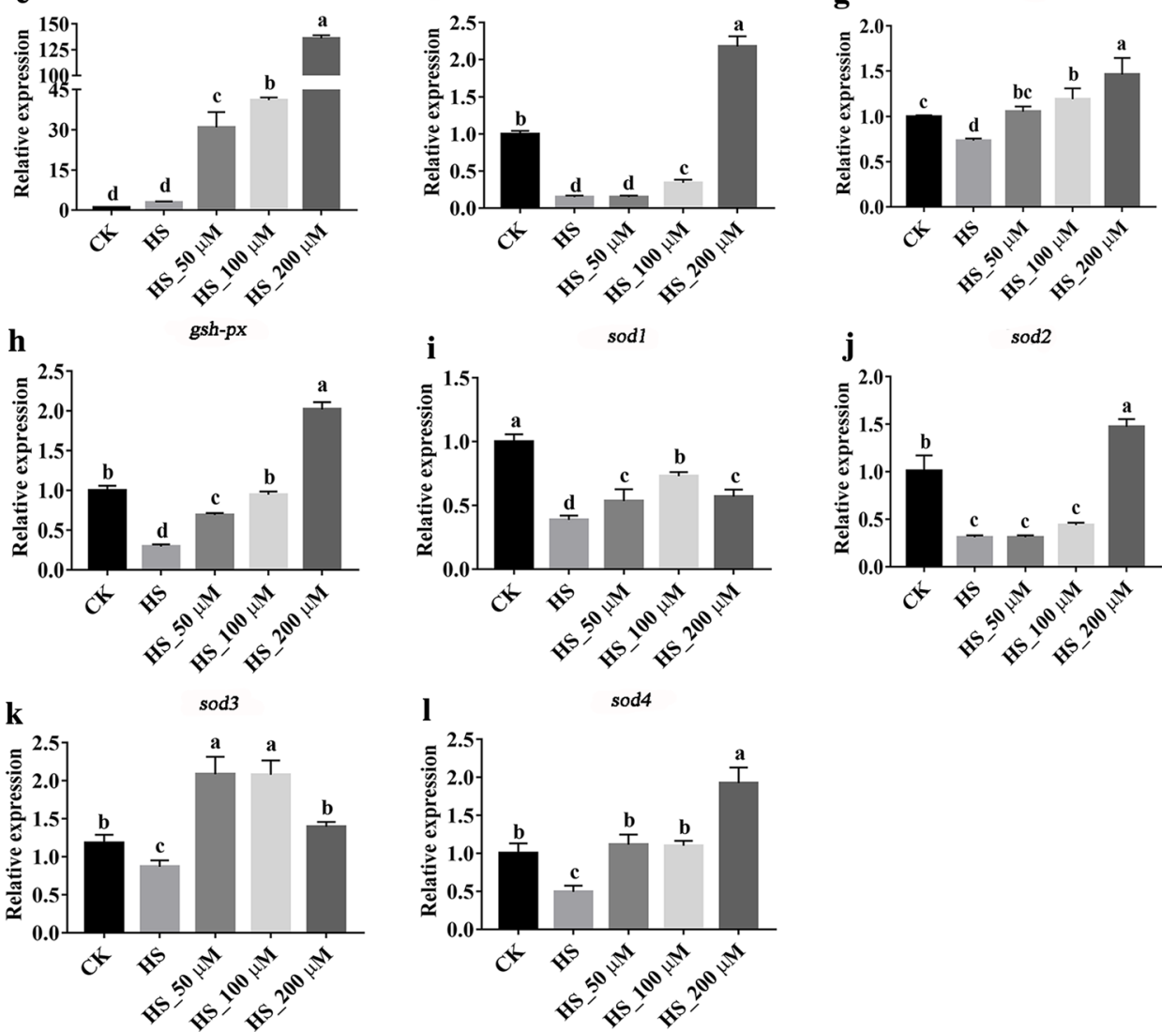

Fig. 9 (See legend on previous page.) 
to further explore the response mechanism through which NO alleviates mycelial damage under HS. A total of 579 DEGs specifically regulated by NO were identified. These genes might participate in the response of P. ostreatus mycelia to HS by regulating cell metabolism, affecting the composition and structure of the cell membrane, or modifying the catalytic activity of proteins. Interestingly, the functional enrichment analysis identified 6 DEGs among the 579 DEGs that were not only regulated by NO but also closely related to the respiratory chain. Among these 6 DEGs, mitochondrial chaperone BCS1 (g12952) and the succinate dehydrogenase iron-sulfur subunit (g3097) are necessary for complexes II and III, respectively, which are closely related to the cytochrome pathway, and AOX (g11376) is involved in the alternative oxidation pathway. Further analysis showed that only AOX was upregulated after SNP addition, whereas the other DEGs were downregulated. In P. ostreatus, NO can induce aox gene expression by inhibiting the expression of aconitase gene and protein, which results in citric acid accumulation [20]. This finding is consistent with our previous results. In plants, it has also been reported that NO can regulate the expression of the aox gene. For instance, Fu et al. showed that NO is an inducer of aox in tobacco plants infected with tobacco mosaic virus [62], and another study found that aox is induced in Arabidopsis cell suspensions treated with NO [63]. Therefore, NO can induce $a o x$ gene expression in both fungi and plants.

AOXs have been documented to occur in plants [64], algae [65], yeasts and pathogenic fungi, such as A. fumigatus [66], Histoplasma capsulatum [67], and Paracoccidioides brasiliensis [68]. AOX is a nonenergy-conserving terminal oxidase in the mitochondrial ETC, and previous studies have shown that AOX functions to balance energy stability and keep the ETC flowing through mitochondria by limiting the formation of mitochondrial ROS $[35,36]$. In plants, aox plays important roles in countering abiotic stresses, including drought, light, temperature and salinity [32, 69, 70]. For example, in tobacco, under drought stress, the recoverability of aox knockdown plants is strongly compromised [71]. In fungi, it has been reported that $a o x$ is upregulated under mitochondrial respiratory chain inhibition or oxidative stress conditions [67, 72]. For example, in P. brasiliensis, aox expression is also upregulated by ROS generation and mitochondrial respiratory chain inhibitors, and heterologous expression of aox in S. cerevisiae decreases intracellular ROS generation [68]. In the presence of $\mathrm{O}_{2}{ }^{-}$, aox in A. fumigatus may play a role in antioxidant defense mechanisms [73]. In this study, the OE-aox strains rapidly resumed their growth after HS, and aox OE could reduce the contents of NADH and ATP and the content and production rate of $\mathrm{O}_{2}^{-}$. In contrast, the opposite results were obtained with the RNAi-aox strains. Previous studies with Arabidopsis thaliana have shown that under hypoxic stress, aox can regulate energy metabolism, affect ROS production and enhance resistance [74], and we obtained similar results. The formation of ROS is mainly due to "single electron leakage" of respiratory chain components, and complexes I and III are considered the main sites of this electron leakage [75]. Therefore, it can be speculated that the aox gene might reduce the production of $\mathrm{O}_{2}{ }^{-}$by regulating the respiratory pathway and maintaining mitochondrial homeostasis and function in P. ostreatus.

A large number of studies have shown that AOX mediates a retrograde signaling pathway that in turn regulates gene expression both transcriptionally and post transcriptionally in response to stress. AOX serves as a link between metabolic activities (mitochondria) and signaling (nucleus) $[41,76]$. In this study, the expression of antioxidant enzyme-encoding genes in the OE-aox strains was downregulated after HS, whereas the expression of these genes in the RNAi-aox strains was upregulated, and these findings might be related to the regulation of ROS production by aox. BHAM is an inhibitor of AOX, and exogenous BHAM also promoted the expression of antioxidant enzyme-encoding genes under HS, which was very similar to the results obtained with the RNAi-aox strains. Therefore, it can be speculated that in response to HS, the aox gene can mediate the retrograde signaling pathway in $P$. ostreatus mycelia to regulate the expression of antioxidant enzyme-encoding genes.

\section{Conclusions}

In summary, our data indicate that $\mathrm{NO}$ responds to HS by reducing the production and accumulation of ROS in P. ostreatus mycelia and can induce $a o x$ gene expression. The construction of $\mathrm{OE}-a \circ x$ and RNAi-aox strains showed that high aox gene expression can affect cell respiration by reducing the content of NADH, which is a product of the TCA cycle, thereby reducing the production and accumulation of ROS. Further studies showed that under HS, aox can mediate the reverse signaling pathway to regulate the expression of antioxidant enzyme genes in $P$. ostreatus, thereby regulating the mycelial response to ROS. Based on these findings, we proposed a potential cascade of cellular events comprising the NO-mediated alleviation of ROS production via aox gene expression (Fig. 10). Our study improves the understanding of the biological functions and regulatory pathways of NO in $P$. ostreatus under HS and provides new ideas for further studies on the functions of AOX in fungi. 


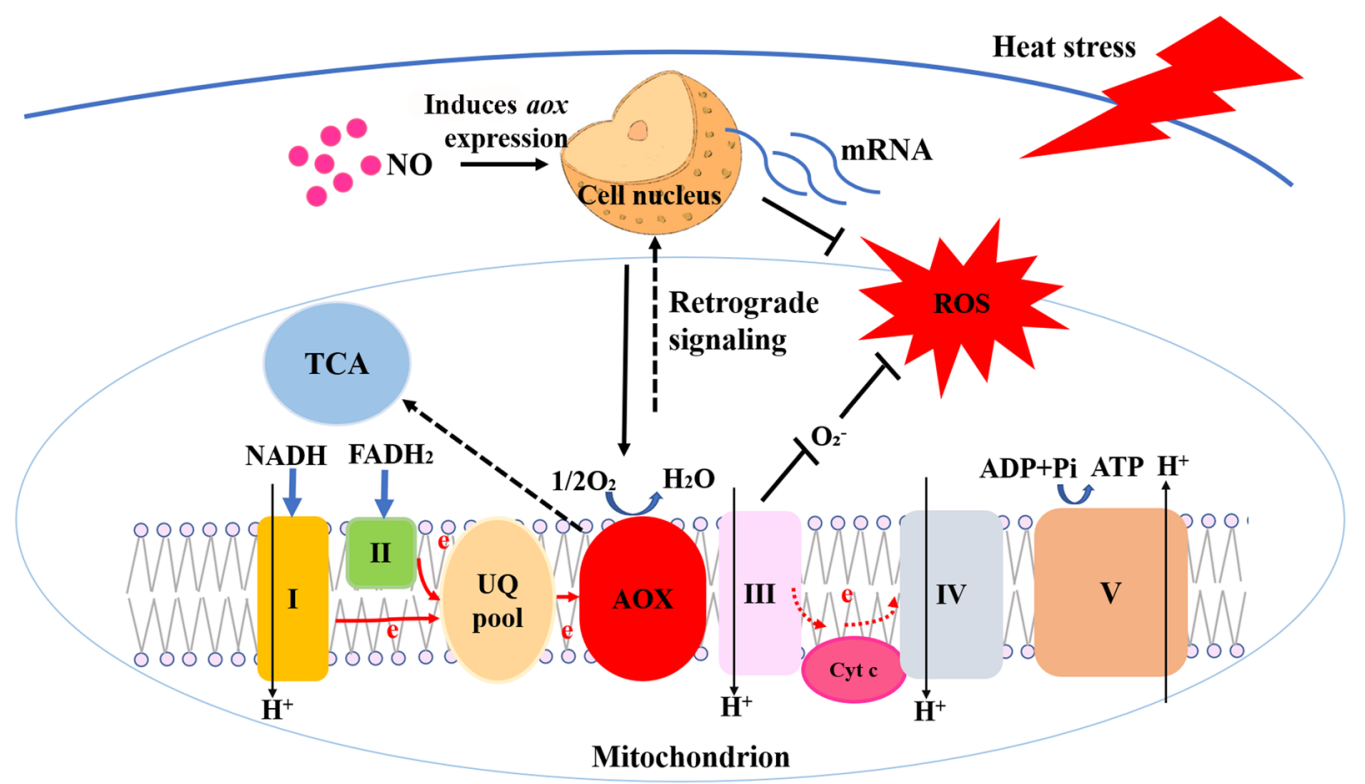

Fig. 10 Schematic representation of the mechanism through which NO alleviates ROS production by inducing aox gene expression under HS. I, II, III, IV, and V: Complexes 1-5, respectively

\section{Methods}

\section{Strains and growth conditions}

The P. ostreatus CCMSSC 00389 strain was provided by the China Center for Mushroom Spawn Standards and Control. The WT, OE-aox, and RNAi-aox strains were incubated on PDA plates. Agrobacterium tumefaciens GV3101 (IMCAS, Beijing, China) was grown in Luria-Bertani (LB) medium (Oxoid, England) containing $100 \mu \mathrm{g} / \mathrm{mL}$ kanamycin (VWR Life Science, USA) and $50 \mu \mathrm{g} / \mathrm{mL}$ rifampin (MP Biomedicals, France) and was used to transform P. ostreatus. Trans 1-T1 phage-resistant chemically competent cells (TransGen Biotech, Beijing, China) were used for plasmid construction and were grown in LB broth containing kanamycin $(50 \mu \mathrm{g} / \mathrm{mL})$.

\section{HS and growth recovery of mycelia}

The WT, OE-aox 47, OE-aox 71, OE-aox 34, RNAiaox 12, RNAi-aox 29 and RNAi-aox 7 strains were used in this study. According to the research methods used in our previous study [4], the different strains were incubated at $28{ }^{\circ} \mathrm{C}$ for $5 \mathrm{~d}$, treated at $40{ }^{\circ} \mathrm{C}$, and then returned to growth at $28{ }^{\circ} \mathrm{C}$. Some of the plates were used to collect mycelia, and the collected mycelia were rapidly placed in liquid nitrogen and stored in a freezer at $-80{ }^{\circ} \mathrm{C}$ for subsequent experiments. After HS, the rest of the strains were placed at $28{ }^{\circ} \mathrm{C}$ to allow them to resume growth, and mycelial growth after 3 and $5 \mathrm{~d}$ was observed and recorded.
For the exogenous BHAM addition experiment described in this paper, the WT strain was cultured at $28{ }^{\circ} \mathrm{C}$ for $5 \mathrm{~d}$ on PDA plates with different concentrations of BHAM (control, $50 \mu \mathrm{M}, 100 \mu \mathrm{M}, 200 \mu \mathrm{M}$ ). Then, the PDA plates of different treatment groups were transferred to $40{ }^{\circ} \mathrm{C}$ for $48 \mathrm{~h}$ and finally placed at $28{ }^{\circ} \mathrm{C}$ to resume growth for $3 \mathrm{~d}$.

\section{Determination of the protein and $\mathrm{H}_{2} \mathrm{O}_{2}$ content}

Protein concentrations were determined using the Bradford Protein Quantification Kit (Vazyme, Nanjing, China), with bovine serum albumin as the standard, following the manufacturer's instructions. The intracellular $\mathrm{H}_{2} \mathrm{O}_{2}$ content was determined using an $\mathrm{H}_{2} \mathrm{O}_{2}$ Quantitative Assay Kit (Sangon Biotech, Shanghai, China) according to the manufacturer's instructions. In an acidic environment, $\mathrm{H}_{2} \mathrm{O}_{2}$ can oxidize $\mathrm{Fe}^{2+}$ to $\mathrm{Fe}^{3+}$, and then $\mathrm{Fe}^{3+}$ combines with dye molecules to form a complex, which has the maximum absorption wavelength at $595 \mathrm{~nm}$. The absorbance at $405 \mathrm{~nm}$ was detected by a microplate reader (Tecan Infinite ${ }^{\circledR}$ M200 Pro, Switzerland) to determine the amount of complex formation, and then the content of $\mathrm{H}_{2} \mathrm{O}_{2}$ was calculated.

\section{Determination of the $\mathrm{O}_{2}^{-}$content and production rate}

Excessive accumulation of $\mathrm{O}_{2}{ }^{-}$will destroy the cell membrane. The content and production rate of $\mathrm{O}_{2}{ }^{-}$in 
the mycelia of the different strains subjected to the different treatments were detected using an $\mathrm{O}_{2}{ }^{-}$Content Detection Kit (Solarbio, Beijing, China) according to the manufacturer's instructions. $\mathrm{O}_{2}{ }^{-}$reacts with hydroxylamine hydrochloride to form nitrite ions, which form a red compound under the action of p-aminobenzenesulfonic acid and naphthalene ethylenediamine hydrochloride, and this compound has a characteristic absorption peak at $530 \mathrm{~nm}$. The content and production rate of $\mathrm{O}_{2}{ }^{-}$in mycelia were then calculated based on the absorbance value at $530 \mathrm{~nm}$ obtained using a microplate reader (Tecan Infinite ${ }^{\circledR}$ M200 Pro, Switzerland).

\section{Determination of the NADH and $\mathrm{NAD}^{+}$content}

Nicotinamide adenine dinucleotide (NAD) is a coenzyme that exists in all cells and is found in two forms: oxidized $\left(\mathrm{NAD}^{+}\right)$and reduced $(\mathrm{NADH})$. In this study, the changes in the $\mathrm{NADH}$ content and the $\mathrm{NAD}^{+} /$ $\mathrm{NADH}$ ratio in the WT and aox-transformed strains under $\mathrm{HS}$ were determined using an $\mathrm{NAD}^{+} / \mathrm{NADH}$ Assay Kit (Beyotime, Shanghai, China) according to the manufacturer's instructions. Ethanol is oxidized to acetaldehyde in the presence of alcohol dehydrogenase, and during this process, $\mathrm{NAD}^{+}$is reduced to NADH. Subsequently, NADH reduces 2-(2-methoxy-4-nitrophenyl)-3-(4-nitrophenyl)-5-(2,4-disulfobenzene) $-2 \mathrm{H}$-tetrazole to orange formazan in the presence of 1-methoxy-5-methylpenazinium methyl sulfate, and the product has a characteristic absorption peak at $450 \mathrm{~nm}$. The samples from the different treatment groups were ground up with liquid nitrogen, and $30 \mathrm{mg}$ was added to $400 \mu \mathrm{L}$ of extraction solution, homogenized, incubated on ice for $10 \mathrm{~min}$, and centrifuged at $12,000 \mathrm{~g}$ and $4{ }^{\circ} \mathrm{C}$ for $10 \mathrm{~min}$. The supernatant was then collected. The total amounts of $\mathrm{NAD}^{+}$and $\mathrm{NADH}$ were determined according to the instructions provided with the kit, and the $\mathrm{NAD}^{+} / \mathrm{NADH}$ ratio was calculated.

\section{Determination of the ATP content and total respiratory rate}

Respiration is the core process of mitochondrial metabolism, and a large amount of free energy is released by oxidative phosphorylation for the production of ATP [77]. In this study, the total respiratory rate was measured according to previous studies [78]. Specifically, the changes in the ATP content during different treatments were measured using an enhanced ATP Assay Kit (Beyotime, Shanghai, China) according to the manufacturer's instructions. This assay is based on the fact that firefly luciferase needs ATP to provide energy for the production of fluorescence. The samples were lysed with the ATP extract in the kit, homogenized for $10 \mathrm{~min}$ and centrifuged at $12,000 \mathrm{~g}$ and $4{ }^{\circ} \mathrm{C}$ for $10 \mathrm{~min}$. The supernatant was then used to determine the ATP content and protein concentration, and the ATP content in different samples was detected by multifunction microplate reader (Tecan Infinite ${ }^{\circledR}$ M200 Pro, Switzerland).

\section{RNA extraction, cDNA library construction, and RNA sequencing (RNA-Seq)}

SNP is a donor of NO, and cPTIO is a scavenger of NO. To understand the regulatory mechanism through which NO alleviates the mycelial damage in $P$. ostreatus induced by HS, four different treatment groups (CK, HS, SNP_HS and cPTIO_HS) were established for RNA-Seq analysis, with 3 replicates in each group and 12 samples in total. For the preparation of RNA-Seq samples described in this paper, the WT strains were cultured at $28{ }^{\circ} \mathrm{C}$ on PDA plates for $5 \mathrm{~d}$ as the CK group and then transferred to new PDA plates with $100 \mu \mathrm{M}$ SNP (SNP_HS) or $250 \mu \mathrm{M}$ cPTIO (cPTIO_HS) treatment according to a previous study [20], after which they were treated with $\operatorname{HS}\left(40{ }^{\circ} \mathrm{C}\right)$ for $48 \mathrm{~h}$. Meanwhile, some WT strains cultured at 28 ${ }^{\circ} \mathrm{C}$ for $5 \mathrm{~d}$ were transferred to the new PDA plates for HS treatment $\left(40^{\circ} \mathrm{C}, 48 \mathrm{~h}\right)$ as the HS group.

Total RNA was extracted from the mycelia of $P$. ostreatus using the $\mathrm{TRIzol}^{\circledR}$ Reagent (Invitrogen, USA), according to the manufacturer's instructions, and genomic DNA was removed using DNase I (TaKaRa, Japan). The RNA quality was determined using a 2100 Bioanalyzer (Agilent, CA, USA). The RNA-Seq transcriptome library was prepared using the $\mathrm{TruSeq}^{\mathrm{TM}}$ RNA sample preparation kit from Illumina (San Diego, CA,USA) and $1 \mu \mathrm{g}$ of total RNA. Briefly, messenger RNA was isolated according to the polyA selection method using oligo (dT) beads and then fragmented using fragmentation buffer. Double-stranded cDNA was then synthesized using a SuperScript double-stranded cDNA synthesis kit (Invitrogen, CA, USA) with random hexamer primers (Illumina). The synthesized cDNA was then subjected to end repair, phosphorylation and ' $\mathrm{A}$ ' base addition according to the library construction protocol established by Illumina. The libraries were subjected to size selection to obtain cDNA target fragments of 200-300 bp using $2 \%$ Low Range Ultra Agarose followed by 15 cycles of PCR amplification with Phusion DNA polymerase (NEB). After quantification by TBS380, the paired-end RNA-Seq library was sequenced with an Illumina NovaSeq 6000 sequencer $(2 \times 150$-bp read length), and the sequencing data were deposited into the Sequence 
Read Archive of the National Centre for Biotechnology Information (NCBI) with the accession number SRP277542.

\section{Analysis of DEGs}

To identify the DEGs between the CK and HS, SNP_ HS and cPTIO_HS groups, the expression level of each transcript was calculated using the fragments per kilobase of exon per million mapped reads (FRKM) method. RSEM (http://deweylab.github.io/RSEM/) was used to quantify the gene abundances [79]. Differential expression analysis was performed using edgeR software in the $\mathrm{R}$ statistical package [80]. In addition, the GO functional enrichment of the DEGs was analyzed.

\section{Construction of RNAi-aox plasmid and strains}

Previous studies have shown that gene knockout and gene transformation using vectors constitute an effective strategy for exploring the function of fungal genes. In addition, OE-aox strains were successfully obtained in our previous study [20]. In this study, the original pCAMBIA1300 vector was modified to harbor the hyg phosphotransferase gene (hyp), which was expressed under the control of the upstream lac promoter. The RNAi-aox plasmid was constructed as follows, the P. ostreatus gpd promoter was PCR amplified, after which the $a o x$-sense and $a o x$-antisence were amplified using primers with homologous arms, as shown in Additional file 1: Table S1. Homologous recombination was then performed to connect the target fragments to the plasmid one by one. The constructed plasmid map is shown in Additional file 1: Fig. S1. The aox-JCF/R primers (Additional file 1: Table S1) were used to amplify the target fragment and thus detect whether the interference plasmid was successfully constructed. Finally, the RNAi-aox plasmid was transformed into $P$. ostreatus mycelia by A. tumefaciens GV3101 according to our previous transformation method [39]. The hyg fragment was amplified to screen the transformed strains, and RT-qPCR was performed to identify the strains with high interference for further experiments.

\section{RNA extraction, reverse transcription and RT-qPCR}

Total RNA was extracted using an E.Z.N.A. Plant RNA Kit (Omega Bio-tek, Norcross, GA, USA) following an extraction method for fungal samples. First-strand cDNA was synthesized using the HiScript II 1st Strand cDNA Synthesis Kit (Vazyme, Nanjing, China) according to the manufacturer's instructions. The ChamQ SYBR RT-qPCR Master Mix Kit (Vazyme, Nanjing, China) and the ABI 7500 real-time PCR amplifier
(Applied Biosystems, Foster City, CA, USA) were used for RT-qPCR. According to our previous study [81], the RT-qPCR amplification procedure was as follows: $95{ }^{\circ} \mathrm{C}$ for $3 \mathrm{~min}, 40$ cycles at $95{ }^{\circ} \mathrm{C}$ for $3 \mathrm{~s}$ and at $60{ }^{\circ} \mathrm{C}$ for $32 \mathrm{~s}$, and a final extension at $72{ }^{\circ} \mathrm{C}$ for $30 \mathrm{~s}$. In this study, RT-qPCR was performed to analyze the mRNA expression levels of the aox gene and key antioxidant enzyme genes in the CCMSSC00389 strain and $a o x$-transformed strains subjected to the different treatments. The $\beta$-actin and $\beta$-tubulin genes were used as internal reference genes, and the relative gene expression was determined according to the $2^{-\triangle \triangle \mathrm{CT}}$ method.

\section{Statistical analysis}

In this study, at least three biological replications were performed in each group. All data were recorded using Excel 2010 software (Microsoft, Redmond, WA, USA). Duncan's multiple range test was used for all significance analysis, and a $P$ value $<0.05$ was considered significant by SPSS software (SPSS Inc., Chicago, IL, USA) [82]. All figures were generated using GraphPad Prism 6 (GraphPad Software Company, San Diego, California, USA).

\section{Abbreviations \\ P. ostreatus: Pleurotus ostreatus; NO: Nitric oxide; aox: Alternative oxidase; HS: Heat stress; TCA: Tricarboxylic acid; ROS: Reactive oxygen species; $\mathrm{Ca}^{2+}$ : Calcium; $\mathrm{H}_{2} \mathrm{O}_{2}$ : Hydrogen peroxide; ETC: Electron transport chain; OE: Overexpression; RNAi: RNA interference; $\mathrm{O}_{2}^{-}$: Superoxide anion; NAD: Nicotinamide adenine dinucleotide; ATP: Adenosine triphosphate; NCBI: National Centre for Biotechnology Information; SNP: Sodium nitroprusside; CPTIO: 2-(4-carboxyphenyl)-4,4,5,5-tetramethylimidazoline-1-oxyl3-oxidec; FRKM: Fragments per kilobase of exon per million mapped reads; DGEs: Differentially expressed genes; GO: Gene ontology; RT-qPCR: Quantitative reverse transcription PCR; RNA-Seq: RNA sequencing; CAT: Catalase; SOD: Superoxide dismutase; TrxR: Thioredoxin reductase; GSH-PX: Glutathione peroxidase; BHAM: Benzohydroxamate.}

\section{Supplementary Information}

The online version contains supplementary material available at https:// doi.org/10.1186/s12934-021-01626-y.

Additional file 1: Fig. S1. Structure of the RNAi-aox vector. Fig. S2 Validation and selection of RNAi-aox strains. A Preliminary selection of RNAi-aox transformed strains by amplification of the hyg gene. B RT-qPCR analysis of the expression of aox in the tested strains. Fig. $\mathbf{S 3}$ Regulation of mycelial growth and aox gene expression by different concentrations of BHAM. A Effects of different concentrations of BHAM on colony morphology. B Effects of different concentrations of BHAM on the mycelial growth rate C Effects of different concentrations of BHAM on aox gene expression in mycelia. The values are presented as the means \pm SEs from three independent experiments. Different letters indicate significant differences among the samples $(P<0.05$ according to Duncan's test).Table S1 Primers used in this study. Table $\mathbf{S 2}$ Reads mapped to the reference P. ostreatus CCMSSC 00389 genome. 


\section{Acknowledgements}

We thank all the members of the research group for their cooperation in the laboratory.

\section{Authors' contributions}

LDH conceived, designed and performed the experiments, analyzed the data, wrote and revised the manuscript. MRZ collaborated in the bioinformatics analysis and in the manuscript writing. QH carried out part of the experiments. $\mathrm{CYH}$ and LJZ designed and revised the manuscript. JXZ conceived and designed the experiments. All authors have read and approved the final manuscript.

\section{Funding}

This work was supported by the China Agriculture Research System of MOF and MARA (CARS-20), the National Basic Research Program of China (No. 2014CB138303) and the Fundamental Research Funds for Central Nonprofit Scientific Institution (No.1610132021029).

\section{Availability of data and materials}

The datasets used and analysed during the current study available from the corresponding author on reasonable request.

\section{Declarations}

\section{Ethics approval and consent to participate}

Not applicable.

\section{Consent for publication}

Not applicable.

\section{Competing interests}

The authors declare that they have no competing interests.

\section{Author details}

${ }^{1}$ Institute of Agricultural Resources and Regional Planning, Chinese Academy of Agricultural Sciences, 10081 Beijing, China. ${ }^{2}$ Key Laboratory of Microbial Resources, Ministry of Agriculture and Rural Affairs, 10081 Beijing, China. ${ }^{3}$ Jilin Agricultural University, 130118 Jilin, China.

Received: 20 August 2020 Accepted: 4 July 2021 Published online: 19 July 2021

\section{References}

1. Jaworska G, Bernas E, Mickowska B. Effect of production process on the amino acid content of frozen and canned Pleurotus ostreatus mushrooms. Food Chem. 2011;125:936-43.

2. Yan ZY, Zhao MR, Wu XL, Zhang JX. Metabolic response of Pleurotus ostreatus to continuous heat stress. Front Microbiol. 2020;10:3148.

3. Song C, Chen Q, Wu XL, Zhang JX, Huang CY. Heat stress induces apoptotic-like cell death in two Pleurotus species. Curr Microbiol. 2014:69:611-6

4. Lei M, Wu XL, Huang CY, Qiu ZH, Zhang JX. Trehalose induced by reactive oxygen species relieved the radial growth defects of Pleurotus ostreatus under heat stress. Appl Microbiol Biotechnol. 2019;103:5379-90.

5. Liu R, Shi L, Zhu T, Yang T, Ren A, Zhu J, Zhao MW. Cross-talk between nitric oxide and calcium-calmodulin regulate ganoderic acid biosynthesis in Ganoderma lucidum under heat stress. Appl Environ Microbiol. 2018;84:e00043-00018.

6. Bellin D, Asai S, Delledonne M, Yoshioka H. Nitric oxide as a mediator for defense responses. Mol Plant Microbe Interact. 2013;26:271-7.

7. Arasimowicz M, Floryszak-Wieczorek J. Nitric oxide as a bioactive signalling molecule in plant stress responses. Plant Sci. 2007;172:876-87.

8. Yoshioka H, Mase K, Yoshioka M, Kobayashi M, Asai S. Regulatory mechanisms of nitric oxide and reactive oxygen species generation and their role in plant immunity. Nitric Oxide. 2010;25:216-21.
9. Peto A, Lehotai N, Feigl G, Tugyi N, Ordog A, Gemes K, Tari I, Erdei L, Kolbert Z. Nitric oxide contributes to copper tolerance by influencing ROS metabolism in Arabidopsis. Plant Cell Rep. 2013;32:1913-23.

10. Singh $R$, Parihar P, Prasad SM. Interplay of calcium and nitric oxide in improvement of growth and arsenic-induced toxicity in mustard seedlings. Sci Rep. 2020;10:6900.

11. Gu RX, Zhu SH, Zhou J, Liu N, Shi JY. Inhibition on brown rot disease and induction of defence response in harvested peach fruit by nitric oxide solution. Eur J Plant Pathol. 2014;139:369-78.

12. Song LL, Ding W, Zhao MG, Sun BT, Zhang LX. Nitric oxide protects against oxidative stress under heat stress in the calluses from two ecotypes of reed. Plant Sci. 2006;171:449-58.

13. Peto A, Lehotai N, Lozano-Juste J, Leon J, Tari I, Erdei L, Kolbert Z. Involvement of nitric oxide and auxin in signal transduction of copper-induced morphological responses in Arabidopsis seedlings. Ann Bot. 2011;108:449-57.

14. Baidya S, Cary JW, Grayburn WS, Calvo AM. Role of nitric oxide and flavohemoglobin homolog genes in Aspergillus nidulans sexual development and mycotoxin production. Appl Environ Microbiol. 2011;77:5524-8.

15. Prats E, Carver TL, Mur LA. Pathogen-derived nitric oxide influences formation of the appressorium infection structure in the phytopathogenic fungus Blumeria graminis. Res Microbiol. 2008;159:476-80.

16. Gong $X Y$, Fu YP, Jiang DH, Li GQ, Yi XH, Peng YL. L-arginine is essential for conidiation in the filamentous fungus Coniothyrium minitans. Fungal Genet Biol. 2007;44:1368-79.

17. Dong JF, Zhang M, Lu L, Sun LN, Xu MJ. Nitric oxide fumigation stimulates flavonoid and phenolic accumulation and enhances antioxidant activity of mushroom. Food Chem. 2012;135:1220-5.

18. Kong WW, Huang CY, Chen Q, Zou YJ, Zhang JX. Nitric oxide alleviates heat stress-induced oxidative damage in Pleurotus eryngii vartuoliensis. Fungal Genetics Biol. 2012;49:15-20.

19. Yu Y, Yang ZJ, Guo K, Li Z, Zhou HZ, Wei YL, Li JS, Zhang XJ, Harvey P, Yang HT. Oxidative damage induced by heat stress could be relieved by nitric oxide in Trichoderma harzianum LTR-2. Curr Microbiol. 2015;70:618-22.

20. Hou LD, Zhao MR, Huang CY, Wu XL, Zhang JX. Nitric oxide improves the tolerance of Pleurotus ostreatus to heat stress by inhibiting mitochondrial aconitase. Appl Environ Microbiol. 2020;86:e02303-19.

21. Rogov AG, Zvyagilskaya RA. Physiological role of alternative oxidase (from yeasts to plants). Biochemistry. 2015;80:400-7.

22. Siedow JN, Umbach AL. The mitochondrial cyanide-resistant oxidase: structural conservation amid regulatory diversity. Biochem Biophys Acta. 2000;1459:432-9.

23. Chae MS, Lin CC, Kessler KE, Nargang CE, Tanton LL, Hahn LB, Nargang FE. Identification of an alternative oxidase induction motif in the promoter region of the aod-1 gene in Neurospora crassa. Genetics. 2007;175:1597-606.

24. Zhang ZL, Huang L, Shulmeister VM, Chi Y, Kim KK, Hung LW, Croftsk AR, Berry EA, Kim SH. Electron transfer by domain movement in cytochrome bc1. Nature. 1998;392:677-84.

25. Tanton LL, Nargang CE, Kessler KE, Li Q, Nargang FE. Alternative oxidase expression in Neurospora crassa. Fungal Genet Biol. 2003;39:176-90

26. Del-Saz NF, Ribas-Carbo M, Mcdonald AE, Lambers H, Fernie AR, Florez-Sarasa I. An in vivo perspective of the role(s) of the alternative oxidase pathway. Trends Plant Sci. 2018;23:206-19.

27. Maxwell DP, Wang Y, Mcintosh L. The alternative oxidase lowers mitochondrial reactive oxygen production in plant cells. PNAS. 1999:96:8271-6.

28. Popov VN. Possible role of free oxidation processes in the regulation of reactive oxygen species production in plant mitochondria. Biochem Soc Trans. 2003;31:1316-7.

29. Rhoads DM, Subbaiah CC. Mitochondrial retrograde regulation in plants. Mitochondrion. 2007;7:177-94.

30. Vanlerberghe GC, Martyn GD, Dahal K. Alternative oxidase: a respiratory electron transport chain pathway essential for maintaining photosynthetic performance during drought stress. Physiol Plant. 2016:157:322-37. 
31. Jian W, Zhang DW, Zhu F, Wang SX, Pu XJ, Deng XG, Luo SS, Lin HH. Alternative oxidase pathway is involved in the exogenous SNP-elevated tolerance of Medicago truncatula to salt stress. J Plant Physiol. 2016;193:79-87.

32. Borovik OA, Grabelnych OI. Mitochondrial alternative cyanideresistant oxidase is involved in an increase of heat stress tolerance in spring wheat. J Plant Physiol. 2018;231:310-7.

33. Luevano Martinez LA, Caldeira da Silva CC, Nicastro GG, Schumacher RI, Kowaltowski AJ, Gomes SL. Mitochondrial alternative oxidase is determinant for growth and sporulation in the early diverging fungus Blastocladiella emersonii. Fungal Biol. 2019;123:59-65.

34. Magnani T, Soriani FM, Martins VdP, Policarpo ACdF, Sorgi CA, Faccioli $\mathrm{LH}$, Curti C, Uyemura SA. Silencing of mitochondrial alternative oxidase gene of Aspergillus fumigatus enhances reactive oxygen species production and killing of the fungus by macrophages. J Bioenerg Biomembr. 2008;40:631-6.

35. Vanlerberghe GC. Alternative oxidase: a mitochondrial respiratory pathway to maintain metabolic and signaling homeostasis during abiotic and biotic stress in plants. Int J Mol Sci. 2013;14:6805-47.

36. Mcintosh $V$. Signals regulating the expression of the nuclear gene encoding alternative oxidase of plant mitochondria. Plant Physiol. 1996;111:589-95.

37. Fouquerel E, Sobol RW. ARTD1 (PARP1) activation and NAD ${ }^{+}$in DNA repair and cell death. DNA Repair. 2014;23:27-32.

38. Tamai S, lida H, Yokota S, Sayano T, Kiguchiya S, Ishihara N, Hayashi Jl, Mihara K, Oka T. Characterization of the mitochondrial protein LETM1, which maintains the mitochondrial tubular shapes and interacts with the AAA-ATPase BCS1L. J Cell Sci. 2008;121:2588-600.

39. Hou LD, Wang LN, Wu XL, Gao W, Zhang JX, Huang CY. Expression patterns of two pal genes of Pleurotus ostreatus across developmental stages and under heat stress. BMC Microbiol. 2019;19:231.

40. Yao X, Li JJ, Liu JP, Liu K. An Arabidopsis mitochondria-localized RRL protein mediates abscisic acid signal transduction through mitochondrial retrograde regulation involving ABI4. J Exp Bot. 2015:66:6431-45.

41. Saha B, Borovskii G, Panda SK. Alternative oxidase and plant stress tolerance. Plant Signal Behav. 2016;11:e1256530.

42. Li H, Chang JJ, Zheng JX, Dong YC, Liu QY, Yang XZ, Wei CH, Zhang Y, Ma JX, Zhang X. Local melatonin application induces cold tolerance in distant organs of Citrullus lanatus L. via long distance transport. Sci Rep. 2017;7:40858.

43. Qu JB, Zhao MY, Tom H, Feng X, Zhang JX, Huang CY. Identification and characterization of small noncoding RNAs in genome sequences of the edible fungus Pleurotus ostreatus. Biomed Res Int. 2016;2016:1-9.

44. Schonbaum G, Bonner W, Storey B, Bahr J. Specific inhibition of the cyanide-insensitive respiratory pathway in plant mitochondria by hydroxamic acids. Plant Physiol. 1971;47:124-8.

45. Jarmuszkiewicz W, Sluse-Goffart CM, Vercesi AE, Sluse FE. Alternative oxidase and uncoupling protein: thermogenesis versus cell energy balance. Biosci Rep. 2001;21:213-22.

46. Lorenzo L, Carlos G-M, Magdalena G, Gabriela P. Nitric oxide: the versatility of an extensive signal molecule. Annu Rev Plant Biol. 2003;54:109-36.

47. Hasanuzzaman M, Nahar K, Hossain MS, Anee TI, Parvin K, Fujita M. Nitric oxide pretreatment enhances antioxidant defense and glyoxalase systems to confer PEG-induced oxidative stress in rapeseed. J Plant Interact. 2017;12:323-31.

48. Rahimian Boogar A, Salehi H, Jowkar A. Exogenous nitric oxide alleviates oxidative damage in turfgrasses under drought stress. South African J Botany. 2014;92:78-82.

49. Domingos P, Prado AM, Wong A, Gehring C, Feijo JA. Nitric oxide: a multitasked signaling gas in plants. Mol Plant. 2015;8:506-20.

50. Wahid A, Gelani S, Ashraf M, Foolad M. Heat tolerance in plants: an overview. Environ Exp Bot. 2007;61:199-223.

51. Moller IM. Plant mitochondria and oxidative stress: electron transport, NADPH turnover, and metabolism of reactive oxygen species. Annu Rev Plant Physiol Plant Mol Biol. 2001;52:561-91.

52. Sharma P, Jha AB, Dubey RS, Pessarakli M. Reactive oxygen species, oxidative damage, and antioxidative defense mechanism in plants under stressful conditions. Journal of Botany. 2012;2012:1-26.
53. Mittler R, Finka A, Goloubinoff P. How do plants feel the heat? Trends Biochem Sci. 2012;37:118-25.

54. Liu R, Zhu T, Yang T, Yang ZY, Ren A, Shi L, Zhu J, Yu HS, Zhao MW. Nitric oxide regulates ganoderic acid biosynthesis by the S-nitrosylation of aconitase under heat stress in Ganoderma lucidum. Environ Microbiol. 2020;23(2).

55. Karpets YV, Kolupaev YE, Yastreb TO. Effect of sodium nitroprusside on heat resistance of wheat coleoptiles: dependence on the formation and scavenging of reactive oxygen species. Russ J Plant Physiol. 2011;58:1027-33.

56. Song LL, Yue LL, Zhao HQ, Hou MF. Protection effect of nitric oxide on photosynthesis in rice under heat stress. Acta Physiol Plant. 2013:35:3323-33.

57. Almeida B, Buttner S, Ohlmeier S, Silva A, Mesquita A, SampaioMarques B, Nuno S, Osório NS, Kollau A, Mayer B, Leão C, Laranjinha J, Rodrigues F, Madeo F, Ludovico P. NO-mediated apoptosis in yeast. J Cell Sci. 2007;120:3279-88.

58. Nasuno R, Aitoku M, Manago Y, Nishimura A, Sasano Y, Takagi H. Nitric oxide-mediated antioxidative mechanism in yeast through the activation of the transcription factor Mac1. PLoS ONE. 2014;9:e113788.

59. Hirayama T, Shinozaki K. Research on plant abiotic stress responses in the post-genome era: past, present and future. Plant J. 2010;61:1041-52.

60. Wu ZJ, Li XH, Liu ZW, Li H, Wang YX, Zhuang J. Transcriptome-based discovery of AP2/ERF transcription factors related to temperature stress in tea plant (Camellia sinensis). Funct Integr Genomics. 2015;15:741-52.

61. Tang S, Dong Y, Liang D, Zhang ZJ, Ye CY, Shuai P, Han X, Zhao Y, Yin W, Xia XL. Analysis of the drought stress-responsive transcriptome of black cottonwood (Populus trichocarpa) using deep RNA sequencing. Plant Mol Biol Reporter. 2014;33:424-38.

62. Fu LJ, Shi K, Gu M, Zhou YH, Dong DK, Liang WS, Song FM, Yu JQ. Systemic induction and role of mitochondrial alternative oxidase and nitric oxide in a compatible tomato-Tobacco mosaic virus interaction. Mol Plant Microbe Interact. 2010;23:39-48.

63. Huang $X$, von Rad U, Durner J. Nitric oxide induces transcriptional activation of the nitric oxide-tolerant alternative oxidase in Arabidopsis suspension cells. Planta. 2002;215:914-23.

64. Borecky J, Vercesi AE. Plant uncoupling mitochondrial protein and alternative oxidase: energy metabolism and stress. Biosci Rep. 2005;25:271-86.

65. Tischner R, Planchet E, Kaiser WM. Mitochondrial electron transport as a source for nitric oxide in the unicellular green alga Chlorella sorokiniana. FEBS Lett. 2004;576:151-5.

66. Tudella VG, Curti C, Soriani FM, Santos AC, Uyemura SA. In situ evidence of an alternative oxidase and an uncoupling protein in the respiratory chain of Aspergillus fumigatus. Int J Biochem Cell Biol. 2003;36:162-72.

67. Johnson CH, Prigge JT, Warren AD, Mcewen JE. Characterization of an alternative oxidase activity of Histoplasma capsulatum. Yeast. 2003;20:381-8

68. Martins VP, Dinamarco TM, Soriani FM, Tudella VG, Oliveira SC, Goldman GH, Curti C, Uyemura SA. Involvement of an alternative oxidase in oxidative stress and mycelium-to-yeast differentiation in Paracoccidioides brasiliensis. Eukaryot Cell. 2011;10:237-48.

69. Sun YJ, Liu XH, Zhai H, Gao HY, Yao YX, Du YP. Responses of photosystem II photochemistry and the alternative oxidase pathway to heat stress in grape leaves. Acta Physiol Plant. 2016;38:232.

70. Analin B, Mohanan A, Bakka K, Challabathula D. Cytochrome oxidase and alternative oxidase pathways of mitochondrial electron transport chain are important for the photosynthetic performance of pea plants under salinity stress conditions. Plant Physiol Biochem. 2020;154:248-59.

71. Wang J, Vanlerberghe GC. A lack of mitochondrial alternative oxidase compromises capacity to recover from severe drought stress. Physiol Plant. 2013;149:461-73.

72. Joseph-Horne T, Hollomon DW, Wood PM. Fungal respiration: a fusion of standard and alternative components. Biochimica et Biophysica Acta. 2001;1504:179-95.

73. Magnani T, Soriani FM, Martins VP, Nascimento AM, Tudella VG, Curti C, Uyemura SA. Cloning and functional expression of the 
mitochondrial alternative oxidase of Aspergillus fumigatus and its induction by oxidative stress. FEMS Microbiol Lett. 2007;271:230-8.

74. Vishwakarma A, Kumari A, Mur LAJ, Gupta KJ. A discrete role for alternative oxidase under hypoxia to increase nitric oxide and drive energy production. Free Radic Biol Med. 2018;122:40-51.

75. Poyton RO, Ball KA, Castello PR. Mitochondrial generation of free radicals and hypoxic signaling. Trend Endocrinol Metab. 2009;20:332-40.

76. Giraud E, Van Aken O, Ho LH, Whelan J. The transcription factor ABI4 is a regulator of mitochondrial retrograde expression of alternative oxidase1a. Plant Physiol. 2009;150:1286-96.

77. Rustin P, Jacobs HT. Respiratory chain alternative enzymes as tools to better understand and counteract respiratory chain deficiencies in human cells and animals. Physiol Plant. 2009;137:362-70.

78. Zhang RY, Hu DD, Zhang YY, Goodwin PH, Huang CY, Chen Q, Gao W, Wu XL, Zou YJ, Qu JB, Zhang JX. Anoxia and anaerobic respiration are involved in "spawn-burning" syndrome for edible mushroom Pleurotus eryngii grown at high temperatures. Sci Hortic. 2016;199:75-80.
79. Li B, Dewey CN. RSEM: accurate transcript quantification from RNA-Seq data with or without a reference genome. BMC Bioinform. 2011;12:323.

80. Robinson MD, Mccarthy DJ, Smyth GK. edgeR: a Bioconductor package for differential expression analysis of digital gene expression data. Biogeosciences. 2010;26:139-40.

81. Wang LN, Wu XL, Gao W, Zhao MR, Zhang JX, Huang CY. Differential expression patterns of Pleurotus ostreatus catalase genes during developmental stages and under heat stress. Genes. 2017:8:335.

82. Norris G, Qureshi F, Howitt D, Cramer D. Introduction to statistics with SPSS for social science. Routledge. 2013.

\section{Publisher's Note}

Springer Nature remains neutral with regard to jurisdictional claims in published maps and institutional affiliations.
Ready to submit your research? Choose BMC and benefit from:

- fast, convenient online submission

- thorough peer review by experienced researchers in your field

- rapid publication on acceptance

- support for research data, including large and complex data types

- gold Open Access which fosters wider collaboration and increased citations

- maximum visibility for your research: over 100M website views per year

At BMC, research is always in progress.

Learn more biomedcentral.com/submissions 